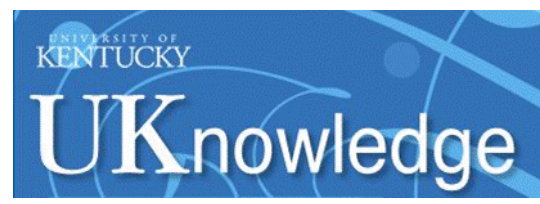

University of Kentucky

UKnowledge

\title{
Clinical Applications of Near-Infrared Diffuse Correlation Spectroscopy and Tomography for Tissue Blood Flow Monitoring and Imaging
}

Yu Shang

North University of China, China

Ting Li

University of Electronic Science \& Technology of China, China

Guoqiang Yu

University of Kentucky, gyu2@uky.edu

Follow this and additional works at: https://uknowledge.uky.edu/cbme_facpub

Part of the Biomedical Engineering and Bioengineering Commons, and the Physiology Commons

Right click to open a feedback form in a new tab to let us know how this document benefits you.

\section{Repository Citation}

Shang, Yu; Li, Ting; and Yu, Guoqiang, "Clinical Applications of Near-Infrared Diffuse Correlation Spectroscopy and Tomography for Tissue Blood Flow Monitoring and Imaging" (2017). Biomedical Engineering Faculty Publications. 38.

https://uknowledge.uky.edu/cbme_facpub/38

This Review is brought to you for free and open access by the Biomedical Engineering at UKnowledge. It has been accepted for inclusion in Biomedical Engineering Faculty Publications by an authorized administrator of UKnowledge. For more information, please contact UKnowledge@lsv.uky.edu. 


\title{
Clinical Applications of Near-Infrared Diffuse Correlation Spectroscopy and Tomography for Tissue Blood Flow Monitoring and Imaging
}

\author{
Digital Object Identifier (DOI) \\ https://doi.org/10.1088/1361-6579/aa60b7 \\ Notes/Citation Information \\ Published in Physiological Measurement, v. 38, no. 4, p. R1-R26. \\ (c) 2017 Institute of Physics and Engineering in Medicine
}

After a 12-month embargo period from the publication of the Version of Record of this article, everyone is permitted to use, copy, and redistribute this article for non-commercial purposes only, provided that they adhere to all the terms of the Creative Commons Attribution-NonCommercial-NoDerivs 3.0 Unported license: https://creativecommons.org/licences/by-nc-nd/3.0

The document available for download is the authors' post-peer-review final draft of the article. 
TOPICAL REVIEW

\section{Clinical applications of near-infrared diffuse correlation spectroscopy and tomography for tissue blood flow monitoring and imaging}

To cite this article: Yu Shang et al 2017 Physiol. Meas. 38 R1

\section{Manuscript version: Accepted Manuscript}

Accepted Manuscript is "the version of the article accepted for publication including all changes made as a result of the peer review process, and which may also include the addition to the article by IOP Publishing of a header, an article ID, a cover sheet and/or an 'Accepted

Manuscript' watermark, but excluding any other editing, typesetting or other changes made by IOP Publishing and/or its licensors"

This Accepted Manuscript is (c) (c) 2017 Institute of Physics and Engineering in Medicine.

During the embargo period (the 12 month period from the publication of the Version of Record of this article), the Accepted Manuscript is fully protected by copyright and cannot be reused or reposted elsewhere.

As the Version of Record of this article is going to be / has been published on a subscription basis, this Accepted Manuscript is available for reuse under a CC BY-NC-ND 3.0 licence after the 12 month embargo period.

After the embargo period, everyone is permitted to use copy and redistribute this article for non-commercial purposes only, provided that they adhere to all the terms of the licence https://creativecommons.org/licences/by-nc-nd/3.0

Although reasonable endeavours have been taken to obtain all necessary permissions from third parties to include their copyrighted content within this article, their full citation and copyright line may not be present in this Accepted Manuscript version. Before using any content from this article, please refer to the Version of Record on IOPscience once published for full citation and copyright details, as permissions will likely be required. All third party content is fully copyright protected, unless specifically stated otherwise in the figure caption in the Version of Record.

View the article online for updates and enhancements. 
2

3

4

5

6

7

8

9

10

11

12

\section{Clinical Applications of Near-infrared Diffuse Correlation Spectroscopy and} Tomography for Tissue Blood Flow Monitoring and Imaging

Yu Shang ${ }^{1}$, Ting $\mathrm{Li}^{2}$, and Guoqiang $\mathrm{Yu}^{3, *}$

${ }^{1}$ Key Laboratory of Instrumentation Science \& Dynamic Measurement

North University of China

No.3 Xueyuan Road

Taiyuan, Shanxi 030051, China

E-mail: yushang@ nuc.edu.cn

${ }^{2}$ State Key Lab Elect Thin Film \& Integrated Device, University of Electronic Science \& Technology of China

Chengdu, Sichuan 610054, China

E-mail: liting@uestc.edu.cn

${ }^{3}$ Department of Biomedical Engineering

University of Kentucky

514C RMB, 143 Graham Avenue

Lexington, KY 40506-0108, USA

*E-mail:guoqiang.yu@uky.edu 


\begin{abstract}
Blood flow is one such available observable promoting a wealth of physiological insight both individually and in combination with other metrics. Near-infrared diffuse correlation spectroscopy (DCS) and, to a lesser extent, diffuse correlation tomography (DCT), have increasingly received interest over the past decade as noninvasive methods for tissue blood flow measurements and imaging. DCS/DCT offers several attractive features for tissue blood flow measurements/imaging such as noninvasiveness, portability, high temporal resolution, and relatively large penetration depth (up to several centimeters). This review first introduces the basic principle and instrumentation of DCS/DCT, followed by presenting clinical application examples of DCS/DCT for the diagnosis and therapeutic monitoring of diseases in a variety of organs/tissues including brain, skeletal muscle, and tumor. Clinical study results demonstrate technical versatility of DCS/DCT in providing important information for disease diagnosis and intervention monitoring.
\end{abstract}




\section{$\underline{\text { Table of Contents }}$}

1. Introduction 4

2. DCS/DCT Methods 6

2.1 Diffuse Correlation Spectroscopy (DCS) 6

2.2 Diffuse Correlation Tomography (DCT) 9

2.3 DCS/DCT Instrumentation 11

3. Clinical Application Examples 12

3.1 Brain 13

3.1.1 Diagnosis of cardio-cerebral diseases 13

3.1.2 Therapeutic monitoring of cardio-cerebral diseases 17

3.2 Skeletal muscle 19

3.2.1 Diagnosis of muscular diseases 19

3.2.2 Therapeutic monitoring of muscular diseases 21

\begin{tabular}{ll}
3.3 Tumor & 22 \\
\hline
\end{tabular}

3.3.1 Diagnosis of tumors 22

3.3.2 Therapeutic monitoring of tumor treatments 24

4. Summary and future perspectives 26

Acknowledgments $\quad 30$

References $\quad 31$ 


\section{Introduction}

Near-infrared (NIR) diffuse optical technologies have gained popularity in recent years as simple, fast, continuous, portable, and relatively inexpensive methods for noninvasive quantification of hemodynamics and metabolism in deep tissues up to several centimeters (Vardi and Nini, 2008; Schachner et al., 2008; Bouye et al., 2005; Boas et al., 2001; Ferrari et al., 2011; Jobsis, 1977; Murkin and Arango, 2009; Shuler et al., 2009). One type of NIR technology, namely near-infrared spectroscopy (NIRS) or diffuse optical spectroscopy (DOS), takes advantage of the low absorption spectrum of biological tissues in the NIR range $(650-950 \mathrm{~nm})$ and penetrates deep tissues to detect light absorption by oxy-hemoglobin $\left(\mathrm{HbO}_{2}\right)$ and deoxy-hemoglobin (Hb) in red blood cells (RBCs) (Kim et al., 2005; Strangman et al., 2003). As a result, oxy-, deoxy-, total- hemoglobin concentrations (i.e., $\left[\mathrm{HbO}_{2}\right]$, [Hb], and $\mathrm{THC})$ and tissue blood oxygen saturation $\left(\mathrm{StO}_{2}\right)$ in local tissue microvasculature can be quantified (Liu et al., 1995; Boas et al., 2001; Wolf et al., 2003; Fantini et al., 1995). Over past decades, a variety of NIRS/DOS technologies have been extensively explored to study various diseases affecting blood oxygenation levels in local and regional tissues (Liu et al., 1995; Wolf et al., 2003; Quaresima et al., 2004; Fantini et al., 1995; Patterson et al., 1989; Al-Rawi and Kirkpatrick, 2006). Correspondingly, diffuse optical tomography (DOT) based on NIRS/DOS has also been developed for 3-dimensional (3-D) imaging of oxygenation distributions in organs or regional tissues (Intes et al., 2010; Eggebrecht et al., 2014; Dehghani et al., 2009; Zhang et al., 2013; Arridge and Hebden, 1997; Jermyn et al., 2013).

Another emerging NIR technology, diffuse correlation spectroscopy (DCS), has also been developed to directly measure blood flow variations in deep tissue microvasculature (Boas et 
al., 1995; Maret and Wolf, 1989; Pine et al., 1988; Yu et al., 2005a; Jaillon et al., 2007; Boas, 1996; Durduran, 2004; Choe, 2005; Zhou, 2007; Irwin, 2011; Gurley, 2012; Cheng, 2013; He, 2015; Dong, 2015). DCS uses coherent NIR light to penetrate deep tissues and monitors temporal light intensity fluctuation caused by moving scatterers (primarily RBCs in the microvasculature) to extract a blood flow index (BFI) (Irwin et al., 2011; Cheung et al., 2001). The relative change of blood flow (rBF) is calculated by normalizing BFI to its baseline value before the physiological change occurs. DCS measurements of tissue blood flow changes have been extensively validated against other standards, including power spectral Doppler ultrasound (Yu et al., 2005b), Doppler ultrasound (Buckley et al., 2009; Roche-Labarbe et al., 2010), laser Doppler (Durduran, 2004; Shang et al., 2011a), Xenon computed tomography (Xenon-CT) (Kim et al., 2010), fluorescent microsphere flow measurement (Zhou et al., 2009), and arterial spin labeling magnetic resonance imaging (ASL-MRI) (Yu et al., 2007). In some studies, DCS has been combined with NIRS/DOS in hybrid instruments to simultaneously measure tissue blood flow and oxygenation (Durduran et al., 2004; Yu et al., 2005a; Cheng et al., 2012; Munk et al., 2012; Shang et al., 2012; Gurley et al., 2012), which allows for the derivation of metabolic rate of tissue oxygen consumption.

Despite advances in DCS applicability, there have been limited tomographic imaging realizations. An early contact-measurement based 3-D diffuse correlation tomography (DCT) approach using an optical fiber array was applied to tissue phantoms with a semi-infinite geometry (Boas and Yodh, 1997), but is disadvantaged in vivo due to the irregular geometries of biological tissues. A few noncontact-measurement based DCT examinations have been recently conducted to avoid tissue hemodynamic variations induced by the probe compression 
or the disturbance of sensitive tissue areas (Culver et al., 2003; Huang et al., 2015a; Zhou et al., 2006; Lin et al., 2014; He et al., 2015; Huang et al., 2015b). In these noncontact measurement systems, lenses were positioned between a sample and an optical fiber array connected to the light sources and detection elements. The noncontact DCS/DCT has been successfully applied in examining blood flow distributions in animal models (Zhou et al., 2006; Culver et al., 2003) and human subjects (Li et al., 2013; He et al., 2015; Lin et al., 2012; Huang et al., 2015c; Huang et al., 2015b).

Since NIRS/DOS/DOT technologies for tissue blood oxygenation measurements have been broadly reviewed (Durduran et al., 2010a; Ferrari et al., 2011; Ghosh et al., 2012; Wolf et al., 2007), our review focuses on the DCS/DCT technologies for tissue blood flow measurements in the clinic. We first introduce the principle and instrumentation of DCS/DCT. We then provide typical clinical examples of DCS/DCT for the diagnosis of diseases and therapeutic monitoring of interventions in a variety of organs/tissues such as brain, skeletal muscle, and tumor. Finally, we highlight the limitations of DCS/DCT and point out future perspectives in technology development and clinical applications.

\section{DCS/DCT Methods}

\subsection{Diffuse Correlation Spectroscopy (DCS)}

DCS originates from the concept of "dynamic light scattering (DLS)", a technology that can be used to quantify the motion of moving scatterers in a thin solution through investigating the correlation of light electric field after photons are scattered once by the moving scatterers (i.e., single light scattering) (Brown, 1993; Fletcher, 1976). The extension from single light scattering to multiple light scattering has been explored since 1980s (Maret 
and Wolf, 1987; Pine et al., 1988; Boas et al., 1995). This extension, called diffusing-wave spectroscopy (DWS) (Pine et al., 1988; Li et al., 2005; Jaillon et al., 2006) or diffuse correlation spectroscopy (DCS) (Cheung et al., 2001; Yu et al., 2005a; Gagnon et al., 2008; Boas and Yodh, 1997; Boas et al., 1995), makes it possible to quantify the motion of moving scatterers in a thick sample, such as red blood cells (RBCs) flowing through biological tissues.

The principle and instrumentation of DCS have been described elsewhere (Boas et al., 1995; Cheung et al., 2001; Durduran and Yodh, 2014; Irwin et al., 2011; Yu, 2012b, a; Yu, 2012c). Briefly, a long-coherence laser delivers NIR light via an optical fiber to the tissue (Fig. 1a). Photons entering into the tissue are either absorbed or more dominantly, scattered by static scatterers (e.g., organelles and mitochondria) and dynamic scatterers (mainly moving RBCs). Due to the scattering effect photons may change directions many times while traveling throughout the tissue, resulting in light diffusion. Only a few photons can eventually reach tissue surface and be collected by a single-mode detector fiber placed millimeters or centimeters away from the source fiber. Photons collected by the detector fiber are detected by a single photon-counting avalanche photodiode (APD) to yield light intensity signals (Fig. 1b).

The detected light intensity fluctuates with time (Fig. 1b), which is caused by the motion of moving scatterers (mainly RBCs) inside the tissue volume measured. To quantify the motion of RBCs (i.e., blood flow) the normalized light intensity temporal autocorrelation function $\left(g_{2}(\tau)\right)$ is calculated by a hardware correlator board or a software correlator, which can then be converted to the normalized electric field temporal autocorrelation function $\left(g_{1}(\tau)\right.$, 
Fig. 1c) through the Siegert relation (Rice, 1954):

$$
g_{2}(\vec{r}, \tau)=1+\beta\left|g_{1}(\vec{r}, \tau)\right|^{2}
$$

where $\tau$ is the correlation delay time, $\vec{r}$ is the position vector, and $\beta$ is a parameter which is dependent of laser stability, coherence length, and the number of speckles detected.

The $g_{1}(\tau)$ can be expressed in an integral form (Boas and Yodh, 1997; Li et al., 2013; Pine et al., 1988; Maret and Wolf, 1987)

$$
g_{1}(\tau)=\frac{\left\langle E(0) E^{*}(\tau)\right\rangle}{\left\langle|E(0)|^{2}\right\rangle}=\int_{0}^{\infty} P(s) \exp \left(-\frac{1}{3} k_{0}^{2}<\Delta r^{2}(\tau)>\frac{s}{l^{*}}\right) d s
$$

Here $E(0)$ and $E^{*}(\tau)$ are the scattered light electric field at time 0 and its conjugation at time $\tau$, respectively. $P(s)$ is the normalized distribution of detected photon path length $s, k_{0}$ is the wave vector magnitude of the light in the medium, and $l^{*}$ is the photon random-walk step length, which is equal to $1 / \mu_{s}^{\prime}$ ( $\mu_{s}^{\prime}$ is the medium reduced scattering coefficient).

The unknown $\left\langle\Delta r^{2}(\tau)>\right.$ represents the mean-square-displacement of moving scatterers, which is conventionally difficult to be solved directly using Eq. 2. Alternately, the integral form of autocorrelation function can be converted to a partial differential equation form (Pine et al., 1990). As such, the unnormalized electric field autocorrelation function $G_{1}(\tau)=$ $\left\langle E(0) E^{*}(\tau)>\right.$ satisfies a correlation diffusion equation (Cheung et al., 2001; Boas and Yodh, 1997)

$$
\left(D \nabla^{2}-v \mu_{a}-\frac{1}{3} v \mu_{s}^{\prime} k_{0}^{2}\left\langle\Delta r^{2}(\tau)\right\rangle\right) G_{1}(\vec{r}, \tau)=-v S(\vec{r})
$$

Here, $v$ is the light speed in the medium; $\mu_{a}$ is the medium absorption coefficient; $D \approx v / 3 \mu_{s}^{\prime}$ is the medium photon diffusion coefficient, and $S(\vec{r})$ is the continuous-wave 
isotropic source. The form of $\left\langle\Delta r^{2}(\tau)\right\rangle$ depends on a flow model specifically adopted. For example, a diffuse model, i.e., $\left\langle\Delta r^{2}(\tau)>=6 D_{B} \tau\right.$, has been found to fit experimental data well over a wide range of different tissues (Cheung et al., 2001; Irwin et al., 2011). Here, $D_{B}$ is the effective diffusive coefficient. To account for the fact that not all scatterers are "moving" in the tissue, a factor $\alpha$, representing the ratio of "moving" scatterers to the total number of scatterers, is added to $\left\langle\Delta r^{2}(\tau)>\right.$ (i.e., $\left\langle\Delta r^{2}(\tau)>=6 \alpha D_{B} \tau\right)$. The combined term, $\alpha D_{\mathrm{B}}$, is referred to as blood flow index (BFI) in biological tissues. The $\alpha D_{\mathrm{B}}$ can be extracted by fitting the measured autocorrelation function curve to an analytical solution of the correlation diffusion equation (Eq. 3) under certain geometries (e.g., semi-infinite boundary) (Cheung et al., 2001; Dong et al., 2012a; Roche-Labarbe et al., 2010; Irwin et al., 2011).

\subsection{Diffuse Correlation Tomography (DCT)}

The extension of DCS to DCT is comparable to that of DOS extension to DOT, which images the optical property distribution based on photon diffusion equation and its inverse solutions (i.e., image reconstruction). A DOT instrument typically illuminates tissue and measures the diffusive light leaving the tissue with multiple sources and detectors on the tissue boundary (Intes et al., 2010; Eggebrecht et al., 2014; Dehghani et al., 2009; Zhang et al., 2013). The diffusive propagation of light in tissue can be characterized with photon diffuse equation and parameterized in terms of the unknown $\mu_{a}(\vec{r})$ and $\mu_{s}^{\prime}(\vec{r})$ at different locations ( $\vec{r}$ ) of the measured tissue volume. One can then "invert" the propagation model to recover these unknown parameters. Imaging with DOT has been described in many papers covering computer simulations, phantom tests, and in vivo applications (Franceschini et al., 2006; Durduran et al., 2010a; Eggebrecht et al., 2014; Arridge and Lionheart, 1998; Arridge 
and Hebden, 1997; Jermyn et al., 2013; Dehghani et al., 2009).

Similarly, DCT requires a large number of sources and detectors to measure autocorrelation functions on the tissue boundary. Early DCT approaches rely on analytical solutions of Eq. 3 with the assumption of simple semi-infinite geometries and/or strict heterogeneities (e.g., spherical anomaly) of tissues (Zhou et al., 2006; Culver et al., 2003), which precludes the transition to complex boundaries and imperfect heterogeneities in realistic tissues.

The finite-element-method (FEM) framework has been applied to model light transport in highly diffuse media to resolve the geometry and heterogeneity limitations for DOS/DOT (Arridge and Lionheart, 1998; Arridge and Hebden, 1997; Eggebrecht et al., 2014; Dehghani et al., 2009; Jermyn et al., 2013). For example, NIRFAST (www.nirfast.org) (Dehghani et al., 2009; Jermyn et al., 2013) and TOAST++ (http://web4.cs.ucl.ac.uk/research/vis/toast/) (Schweiger and Arridge, 2014) are publically available image reconstruction toolboxes based on the diffusive model and FEM for DOT

Exploiting the high mathematical similarity of the forward and inverse problems (e.g., boundary conditions and mathematical assumptions) between DOT and DCT, our group first reported the application of FEM in DCT (Lin et al., 2014; Huang et al., 2015a; He et al., 2015; Huang et al., 2015b). We introduced this concept into the FEM-based light transport and image reconstruction modules in NIRFAST (Dehghani et al., 2009), as a shortcut for FEM implementation of DCT. Specifically, we used the "effective $\mu_{a}(\vec{r})$ " (i.e., $\left.\mu_{a}(\vec{r})+2 \mu_{s}^{\prime}(\vec{r}) k_{0}^{2} \Delta \alpha D_{B}(\vec{r}) \tau\right)$ and measured $G_{1}(\vec{r}, \tau)$ to replace the $\mu_{a}(\vec{r})$ and photon fluence rate $\Phi(\vec{r}, t)$ respectively in the NIRFAST for the accomplishment of DCT in tissues 
with complex boundaries (Lin et al., 2014; Huang et al., 2015a; He et al., 2015; Huang et al., 2015b). Computer simulations, phantom tests, and in-vivo studies have verified the accuracy of our modifications of NIRFAST adapted for DCT. As a result, the realm of DCT becomes available for comprehensive imaging under complex geometries and heterogeneous optical properties.

\subsection{DCS/DCT Instrumentation}

Typical DCS/DCT systems are illustrated in Fig. 2. The DCS/DCT systems consist of long-coherence NIR lasers (e.g., 785 nm, Crystalaser, USA), single photon-counting APDs (e.g., SPCM-AQR-12, Perkin Elmer Inc., Canada), and digital correlator boards (e.g., FLEX03LQ, www.correlator.com, USA). A control panel (laptop or desktop) is used to control the DCS/DCT system for data collection and calculation of temporal autocorrelation function via the correlator board. Blood flow index $\left(\alpha D_{\mathrm{B}}\right)$ can be extracted by fitting the measured autocorrelation function curve to an analytical solution of Eq. 3 under certain geometries. In contrast to the portable DCS device (b) with a limited number of sources and detectors, the DCT instrument (c) has a large number of sources and detectors for 3-D flow imaging.

A variety of fiber-optics probes have been designed for DCS (Fig. 3) and DCT (Fig. 4) measurements in different applications (Huang et al., 2015c; Durduran et al., 2005; Yu et al., 2011; Shang et al., 2011b; Huang et al., 2015b) (see Section 3). These probes are connected to the DCS/DCT instruments (Fig. 2) through optical fibers for light delivery and detection. Examples of DCS probes for the studies of brains, skeletal muscles, and tumors are illustrated in Fig. 3a-e, respectively. For instance, a hand-held probe was designed to scan over a breast 
tumor in both horizontal and vertical directions for diagnostic purpose (Durduran et al., 2005)

(Fig. 3d). For the tissues whose shapes promote hemodynamic variations induced by compression or disturb sensitive areas, DCS probes were designed in a noncontact manner (Huang et al., 2015c) (Fig. 3f). In the noncontact measurement design, a lens system with separated source and detector paths was utilized to focus the light from the source and detector fibers on the measured tissue surface. The noncontact DCS (ncDCS) probe was also extended to a noncontact imaging probe head for DCT measurements, which consisted of a linear array of 15 photodetectors and two laser sources connected to a mobile lens-focusing system (Fig. 4) (Lin et al., 2014; He et al., 2015; Huang et al., 2015b). A motorized stage was utilized for automatic scanning (Fig. 4a), enabling large ROI coverage and flexible S-D arrangements without greatly increasing hardware requirements and costs. This new design of noncontact DCT (ncDCT) probe, combined with a novel FEM framework for DCT image reconstruction, has been validated in a tissue-like phantom with anomaly flow contrast design (Fig. 4d) (Lin et al., 2014) and has been applied in detection of breast tumors (He et al., 2015; Huang et al., 2015b). In practice, any innovations adopted in DOS/DOT probes can be adapted for the design of DCS/DCT probes, and a combined probe integrating DOS/DOT and DCS/DCT measurements can be constructed by adding extra optical fibers.

\section{Clinical Application Examples}

DCS/DCT technologies have been extensively explored to be used in animal models and human subjects for the diagnosis and therapeutic monitoring of diseases in various tissues and organs including brains (Cheng et al., 2014; Hou et al., 2014; Busch et al., 2016a; Kim et al., 2014; Kim et al., 2010; Favilla et al., 2014; Lin et al., 2016; Dehaes et al., 2014; Durduran et 
al., 2010b; Shang et al., 2011b; Buckley et al., 2013; Zirak et al., 2014), skeletal muscles (Henry et al., 2015; Mesquita et al., 2013; Yu et al., 2005a; Shang et al., 2012; Gurley et al., 2012), and tumors (Chung et al., 2015; Choe et al., 2014; Zhou et al., 2007; Durduran et al., 2005; He et al., 2015; Sunar et al., 2006; Dong et al., 2012b; Dong et al., 2016). Since this review focuses on the clinical applications, only typical clinical examples are presented in the following subsections based on different types of tissues/organs. For more details, readers are encouraged to read primary papers that are cited but not discussed here and to learn from recent reviews (Yu, 2012a; Yu, 2012c; Durduran and Yodh, 2014; Mesquita et al., 2011).

\subsection{Brain}

\subsubsection{Diagnosis of cardio-cerebral diseases}

DCS has been explored for the diagnosis and evaluation of cardio-cerebral diseases affecting cerebral blood flow (CBF) in adults, children, and neonates. For example, DCS was utilized to investigate $\mathrm{CBF}$ responses in adult patients with cerebral diseases including vasovagal syncope (Cheng et al., 2014), obstructive sleep apnea-hypopnea (Hou et al., 2014; Busch et al., 2016a), traumatic brain injury (Kim et al., 2010; Kim et al., 2014), and ischemic stroke (Durduran et al., 2009; Favilla et al., 2014).

Vasovagal syncope (VVS) is the sudden loss of consciousness, which can be fatal if occurring in public such as driving and flying. In a study of CBF variations to predict VVS (Cheng et al., 2014), a 70-degree head-up-titling (HUT) protocol was applied to 14 healthy adults, and relative changes of $\mathrm{CBF}(\mathrm{rCBF})$ to the baseline were continuously monitored using a fiber-optic probe illustrated in Fig. 3a. In addition, relative changes of main artery blood pressure (rMAP) were also continuously monitored using a noninvasive finger sensor 
(Portapres, FMS Inc., Netherlands). In the subjects having presyncope symptoms during HUT, physiological responses to the tilting were observed to have two stages; while Stage I showed a small changes, Stage II demonstrated a rapid and dramatic decreases in both $\mathrm{rCBF}$ and rMAP that were coincided with presyncope symptoms (Fig. 5). On average, rCBF reached the Stage II earlier and decreased larger $(76 \pm 8 \%$, from a baseline of $100 \%)$ than rMAP $(39 \pm$ $19 \%$, assigning a baseline value of $100 \%$ ) during presyncope. Moreover, a threshold of $\sim 50 \%$ rCBF decline was determined to completely separate the subjects with or without presyncope. This study suggests that continuous monitoring of CBF variations by the portable DCS device may provide predictive information to prevent VVS (e.g., using an automatic feedback to maintain MAP and CBF at normal levels).

Another example of DCS applications is the study of obstructive sleep apnea-hypopnea (OSAH) (Hou et al., 2014), a disease characterized by repetitive pausing of breath resulted from upper airway obstruction during sleep. The impeded airflow during OSAH may cause cerebral ischemia and disturb CBF. Although OSAH can be diagnosed through monitoring of overnight sleep with a polysomnography, evaluation of cerebral ischemia and hypoxia induced by OSAH is not routinely conducted, due to lack of appropriately technologies. Using a dual-wavelength DCS flow-oximeter (Shang et al., 2009), our group conducted continuous and simultaneous measurements of relative changes of CBF, oxy- and deoxy- and total hemoglobin concentrations $\left(\mathrm{rCBF}, \Delta\left[\mathrm{HbO}_{2}\right], \Delta[\mathrm{Hb}], \Delta \mathrm{THC}\right)$ in adult subjects with OSAH for $\sim 8$ hours overnight (Hou et al., 2014). Two fiber-optic probes connected to the DCS flow-oximeter were taped on both sides of the subject's forehead for cerebral monitoring during sleep (illustrated in Fig. 3b). To minimize the disturbance to patients, we remotely 
operated the optical measurements in a control room and designed special fiber-optic connectors which could easily disconnect the probes when the patient went to bathroom. The results showed that apneic events caused significant variations in $\mathrm{rCBF}$ and $\triangle \mathrm{THC}$. Moreover, the degrees of these hemodynamic variations were significantly correlated with the severity of OSAH.

Similarly, DCS was also adopted to measure CBF responses to hypercapnia during wakefulness rather than during sleep in children with obstructive sleep syndrome (OSA) and/or snores (Busch et al., 2016a). Both OSA and snore subjects were found to have significant less changes in $\mathrm{CBF}$ during hypercapnia than health controls, indicating the diseased-induced blunted brain responses.

DCS was also utilized to monitor CBF variations in adults with traumatic brain injury (Kim et al., 2014; Kim et al., 2010) or ischemic stroke (Favilla et al., 2014; Durduran et al., 2009). In those studies, CBF changes were found to be associated with large variations in cerebral blood pressure induced by physiological manipulations (Kim et al., 2010; Favilla et al., 2014; Durduran et al., 2009), indicating the impairments of cerebral autoregulation to maintain a constant $\mathrm{CBF}$.

In addition to the applications in adults and children, DCS was employed to assess CBF in neonates with malfunctions/defects and during surgical treatments, including those with congenital heart disease (CHD) (Durduran et al., 2010b), hypoxic ischemic encephalopathy (Dehaes et al., 2014), single-ventricle CHD undergoing surgery (Dehaes et al., 2015), as well as the neonates during open heart surgery (Busch et al., 2016b). In those studies, DCS 
measurements benefited from higher signal-to-noise ratio (SNR) due to the thinner skulls of neonates.

When subjected to a protocol of $\mathrm{CO}_{2}$ inhalation for inducing hypercapnia (Durduran et al., 2010b), CBF values in neonates with CHD were elevated significantly (158 $\pm 6 \%$, assigning a baseline of 100\%), which were detectable by both DCS and ASL-MRI measurements. Since complex CHD may lead to impaired cerebral autoregulation and low-baseline CBF, this study explored the potential of DCS for longitudinally probing cerebral defects in critically ill neonates.

Apart from physiological challenging mentioned above, CBF values before and after heart surgeries were measured and compared between the neonates with single-ventricle CHD and healthy controls (Dehaes et al., 2015). The results showed impaired cerebral development in diseased neonates, as characterized by decreased CBF. Furthermore, CBF was continuously monitored during neonatal open heart surgery, an intervention aiming to treat heart defects (Busch et al., 2016b). Significant variations in CBF during deep hypothermia, circulatory arrest, and rewarming were observed, indicating the capability of DCS for cerebral hemodynamic monitoring in a highly challenging clinical environment.

In study of cerebral hemodynamics in premature neonates (Roche-Labarbe et al., 2010), a steady increase (28\%, from a baseline of $100 \%)$ in CBF was found over the first six weeks of life, indicating that the neonates gained improvements in brain microcirculation since birth. In another study of extremely preterm neonates, the subjects with hemorrhage were found to have lower CBF when compared with those without hemorrhage (Lin et al., 2016). Similarly, 
lower CBF levels in neonates with hypoxic ischemic encephalopathy were reported, compared to age-matched healthy neonates (Dehaes et al., 2014).

In summary, many cardio-cerebral diseases affect nervous system, cerebral microvasculature, and cerebral oxygen kinetics, leading to abnormality in CBF (Durduran and Yodh, 2014; Mesquita et al., 2011). Therefore, CBF is considered as an important biomarker of brain health, which links the oxygen demand, supply, and consumption. Continuous measurements of $\mathrm{CBF}$ in the clinical setting, however, are not easily achievable due to technology unavailability. DCS offers a continuous, fast, portable, and low-cost tool to noninvasively monitor CBF variations at the bedside of the clinic. Studies presented in this subsection demonstrate that $\mathrm{CBF}$ abnormities are associated with a variety of cerebral diseases. While the explicit relationships between CBF abnormities and clinical outcomes need to be further investigated, these studies support DCS as a useful tool for the diagnosis of cerebral diseases in adults, children, and neonates.

\subsubsection{Therapeutic monitoring of cardio-cerebral diseases}

DCS has been used to evaluate the effects of surgical interventions on CBF in adults and infants. Those surgeries included carotid endarterectomy in adults (Shang et al., 2011b), thrombolysis in adults (Zirak et al., 2014), and cardiac surgery in infants (Buckley et al., 2013).

Carotid endarterectomy (CEA) is a surgical intervention to restore the blood supply to brain through removal of the blockage in carotid artery (Shang et al., 2011b). During CEA, some of major vessels are temporarily clamped, causing a shutdown of blood supply to local cerebral tissues. Thus, intraoperative monitoring of CBF variation is particularly important as 
it permits acute assessment of cerebral hemodynamic level during surgery and post-surgical hemodynamic improvement in the brain. Electroencephalography (EEG) is an often-used technology for cerebral monitoring during CEA, which offers indirect assessment of cerebral ischemia through analysis of brain waves. In a comparison study of EEG and DCS measurements for evaluating cerebral hemodynamic variations during CEA, EEG electrodes were placed all over the scalps of eleven patients undergoing CEA, and two optical probes were taped on both sides of forehead for simultaneous measurements of $\mathrm{CBF}$ and cerebral oxygenation. The internal carotid artery (ICA) clamps during CEA resulted in significant CBF decreases $(-24.7 \pm 7.3 \%$, assigning a baseline of $100 \%)$ at the surgical sides (Fig. 6a). On the other hand, post-CEA CBF values were significantly higher $(+43.2 \pm 16.9 \%$, assigning a baseline of $100 \%$ ) than pre-CEA CBF values. Flow compensation via circle of Willis was also observed at the nonsurgical side (Fig. 6b). CBF responses to ICA clamping were found to be significantly faster, larger, and more sensitive than EEG responses (Fig.7). Simultaneous monitoring of CBF and EEG provided a comprehensive evaluation of cerebral physiological status and showed potential for the adoption of acute interventions (e.g., shunting, medications) during CEA to reduce the risks of severe cerebral ischemia and cerebral hyperperfusion syndrome.

DCS was also applied to a patient with acute ischemic stroke, who received thrombolytic therapy to restore CBF (Zirak et al., 2014). CBF was monitored by DCS throughout the 60-minute thrombolytic therapy. DCS data showed an acute improvement in CBF after thrombolytic therapy, which agreed with the improvement in stroke scale score. This study suggested the potential of continuous DCS monitoring for therapeutic evaluation 
of acute stroke.

DCS measurements of CBF during interventions were also reported in infants. For example, CBF, cerebral oxygenation, and cerebral oxygen metabolism were continuously monitored during the period of postoperative neonatal cardiac surgery using a hybrid optical instrument (Buckley et al., 2013). Due to the complicated communications between the cerebral and cardiac vessels, the surgeries on hearts were found to significantly affect cerebral oxygen extraction fraction, but not $\mathrm{CBF}$ and cerebral metabolic rate of oxygen consumption.

In summary, many intervention strategies for cardio-cerebral diseases are to restore tissue blood flow. Studies presented in this subsection show high sensitivity of DCS for continuous monitoring of $\mathrm{CBF}$ variations during interventions, which holds the potential for dynamic assessment and optimization of cardio-cerebral interventions to maintain CBF in a normal level.

\subsection{Skeletal muscle}

\subsubsection{Diagnosis of muscular diseases}

DCS has been adopted to evaluate the vascular diseases affecting skeletal muscle perfusion such as fibromyalgia and peripheral arterial disease (Shang et al., 2012; Mesquita et al., 2013). Evaluation of muscular diseases was often performed in a dynamic manner because those diseases restrict patients from performing leg or arm exercises. Blood flow responses to exercise reflect how skeletal muscles properly perform physiological functions.

In a study of fibromyalgia (FM) (Shang et al., 2012), women with FM were instructed to perform knee extension exercises with steadily increasing intensity. Calf muscle 
hemodynamics was continuously monitored by a hybrid instrument during exercise using a probe illustrated in Fig. 3c. Results showed that subjects with FM had significantly lower oxygen extraction rate during exercise than healthy controls, and the time of oxygenation recovery were significantly longer. These results suggested an alteration of muscle oxygen utilization in the FM population.

In another study investigating the influence of peripheral arterial disease (PAD) on skeletal muscle hemodynamics (Mesquita et al., 2013), calf blood flow values were found to occur at more depressed levels in PAD population after treadmill and pedal exercises, compared to those in age-matched healthy people. These results indicated adverse impact of PAD on blood flow responses to exercise.

Limitations existed when using DCS techniques to continuously monitor muscle hemodynamic changes during exercise, such as the nature of relative measurement (rBF) and the motion artifact induced by the exercise. To overcome these limitations, our group recently created a calibration protocol for absolute flow measurements and a gating algorithm to minimize motion artifacts during exercise (Gurley et al., 2012; Henry et al., 2015). Briefly, a hybrid DCS/NIRS instrument along with a pre-exercise arterial occlusion protocol was utilized to quantify the absolute baseline blood flow value before exercise. The absolute baseline flow value was then used to calibrate the measured $\mathrm{rBF}$ during exercise in order to obtain absolute flow measurements over the entire exercising period. Motion artifacts were minimized using a novel dynamometer-based gating algorithm embedded in the DCS control software. Essentially, this software determined muscle contraction status based on signals from the dynamometer and recorded data only when muscle fiber motion was minimal. 
The innovative calibration and gating algorithms have been recently applied on patients with FM and compared with age-matched healthy controls. Blood flow responses in forearm muscles to handgrip exercise were continuously monitored and patients with FM exhibited less capability to perform handgrip exercise than healthy controls, evidenced by the weaker blood flow responses during exercise (Fig. 8).

Overall, deficiency of skeletal muscle function may result from mitochondrial dysfunction (Cordero et al., 2010), lower capillary density (Lindh et al., 1995; Morf et al., 2005), reduced capillary permeability (Grassi et al., 1994) and impaired vasodilatory capacity (Kasikcioglu et al., 2006; McIver et al., 2006), all of which are difficult to measure noninvasively. Hybrid DCS/NIRS devices enable noninvasive dynamic quantification of blood flow, blood oxygenation, and oxidative metabolism during exercise, providing unique and comprehensive diagnostic information for skeletal muscle diseases.

\subsubsection{Therapeutic monitoring of muscular diseases}

A few studies have been reported using DCS to investigate the impacts of surgical interventions on skeletal muscle diseases (Yu et al., 2011; Huang et al., 2015c). For muscular revascularization, ultrasound Doppler is routinely used in surgical rooms to test post-surgical reperfusion in large vessels. However, ultrasound Doppler cannot directly assess the restoration of tissue microcirculation, which is generally the major goal of revascularization.

Using a portable DCS flow-oximeter and the probe illustrated in Fig. 3c (Shang et al., 2009), we continuously monitored calf muscle blood flow variations on twelve human limbs undergoing artery revascularization (Yu et al., 2011). The high sensitivity of DCS in detecting blood flow changes was confirmed through clamping or ballooning on/off the 
femoral or aorta arteries during surgeries. Furthermore, immediate hyperemia following the release of occlusions and post-surgery flow improvements in calf muscles were observed, indicating the potential of the DCS for objective assessment of revascularization effects.

Another representative application of DCS is the assessment of blood flow in free transfer muscle flaps following salvage surgeries in patients with head and neck cancer. Conventional approaches to evaluate the success of tissue flaps rely on visual examination along with blood flow measurements in large vessels by ultrasound Doppler. DCS offers a tool to directly assess tissue blood flow in peripheral muscle flaps, which is crucial to evaluate the success of surgeries. For intraoperative monitoring of reconstructive flaps, we designed a lens system which focused the sources and detectors on the tissue surface, permitting noncontact measurements of flap blood flow (see Fig. 3f) (Huang et al., 2015c). The noncontact DCS probe was applied to eight free muscle flaps at multiple time points of blood flow measurements during and post the surgical operations. Fig. 9 shows comparison results obtained from seven successful flaps and one unsuccessful flap. Postoperative blood flow values in the successful flaps were significantly higher than the intraoperative baseline values, indicating a gradual recovery of flap vascularity after the tissue transfer. By contrast, postoperative blood flow recovered much less in the unsuccessful flap. Measurement of blood flow recovery after flap anastomosis holds the potential to act early to salvage ischemic flaps.

\subsection{Tumor}

\subsubsection{Diagnosis of tumors}

The first translational case of DCS for clinical cancer diagnosis was reported on human breast tumors (Durduran et al., 2005). Through scanning over the breast tumors (n = 5) using 
a contact optical probe illustrated in Fig. 3d, higher blood flow contrasts were found in malignant ( $230 \%$ by averaging over three subjects) and benign ( $153 \%$ by averaging over two subjects) tumors, respectively, as compared to the surrounding normal tissues (assigning $100 \%)$. Similar measurement configuration was thereafter applied to a larger population with breast tumors $(\mathrm{n}=32)($ Choe et al., 2014), confirming the high blood flow contrasts in breast tumors (225 227\% in mean value and 190 270\% in 95\% confidence intervals).

Moreover, a recent pilot study investigated the correlations between tumor hemodynamic parameters (i.e., blood flow, $\left[\mathrm{HbO}_{2}\right],[\mathrm{Hb}], \mathrm{StO}_{2}, \mathrm{THC}$ ) and tumor histopathological biomarkers. Increased blood supply to breast tumors was observed, which agreed with the high expression level of Ki67 nuclei in the confirmed breast tumors (Chung et al., 2015). This study suggested that the macroscopic measurements of tissue hemodynamics could reveal pathological properties of breast cancer in microscopic level.

A significant problem with contact DCS measurements described above is the deformation of soft breast tissue, which may distort blood flow distribution in the soft breast. To solve this problem, noncontact DCS/DCT (ncDCS/ncDCT) systems with unique fiber-optic probes were recently developed enabling fully noncontact measurement/imaging of blood distributions in deep tissue volumes with complex boundaries (Lin et al., 2014; Lin et al., 2012; Li et al., 2013; Huang et al., 2015c; He et al., 2015; Huang et al., 2015b). The noncontact probe was attached to a motorized stage that scanned linearly or rotationally over a ROI (Fig. 4). Fig. 10 shows in vivo imaging results from two breast carcinomas. Higher blood flow contrasts (5.9and 10.9-fold) in the tumor regions compared to the surrounding tissues were observed. The reconstructed locations of the two tumors matched ultrasound imaging results when the tumor 
was within the sensitivity region of diffuse light. The ncDCT system showed the promise to image blood flow distributions in soft and vulnerable tissues without distorting tissue hemodynamics.

The autonomic growth and spread of tumors are dependent on increased angiogenesis arising from the increased metabolic demand. Since functional alternations in tumors often appear earlier than detectable morphological changes, functional imaging of tumor blood flow by DCT is a new strategy for early cancer diagnosis and localization.

\subsubsection{Therapeutic monitoring of tumor treatments}

It has been found that the patients with hypoxic tumors show incomplete clinical responses when receiving chemo-radiation therapy that requires tissue oxygen for treatment efficacy (Busch et al., 2000; Carlson, 2006). Many tumors are hypoxic because of abnormal vasculature, malignancy-related anemia, and/or high oxygen consumption by tumor cells. Studies have shown that pretreatment tumor hypoxia is associated with significantly poor responses to the therapy when compared to oxygenated tumors. However, some well-oxygenated tumors failed to respond while some hypoxic tumors responded well, possibly due to the dynamic changes during treatment in tumor oxygen status induced by radiation. Therefore, repeated monitoring of individual tumor hemodynamic status during therapy may provide predictive information for treatment outcomes.

To date, DCS has been utilized to monitor the tumor hemodynamic responses to chemo-radiation therapy in breast tumors (Zhou et al., 2007) and head/neck tumors (Sunar et al., 2006; Dong et al., 2012b; Dong et al., 2016). In a case study of breast cancer using a hybrid DCS/NIRS device, significant changes in tumor blood flow and blood oxygenation 
were observed in the first week of chemo-radiation therapy (Zhou et al., 2007).

In another pilot study of head/neck tumors using a hybrid DCS/NIRS instrument, tumor hemodynamic responses in a small group of patients $(n=8)$ were continually measured once a week over the period of chemo-radiation therapy (Sunar et al., 2006). Tumors exhibited significant dynamic flow and oxygenation changes during the first four weeks of the treatment. This study, however, was limited by the small number of patients examined and only one patient out of 8 showed a partial response to the treatment.

Very recently, our group employed a hybrid DCS/NIRS instrument to continually monitor tumor hemodynamic responses to chemo-radiation therapy for early prediction of treatment outcomes in a relative large patient population with head/neck cancers (Dong et al., 2016). Forty-seven patients were measured once per week over 7 weeks of treatment period to evaluate the hemodynamic status of clinically involved cervical lymph nodes. Patients were classified into two groups: complete response $(\mathrm{CR})(\mathrm{n}=29)$ and incomplete response (IR) (n $=18$ ). Interestingly, tumor hemodynamic responses were found to be associated with clinical outcomes $(\mathrm{CR} / \mathrm{IR})$, wherein the associations differed depending on human papillomavirus (HPV-16) status. In HPV-16 positive patients, significantly lower levels in tumor $\left[\mathrm{HbO}_{2}\right]$ at weeks 1 to 3 , THC at week 3, and $\mathrm{StO}_{2}$ at week 3 were found in the IR group. In HPV-16 negative patients, significantly higher levels in tumor BFI and $\mu_{\mathrm{s}}$ ' at week 3 were observed in the IR group. These hemodynamic parameters exhibited significantly high accuracies for early prediction of clinical outcomes, within the first three weeks of therapy, with the areas under the receiver operating characteristic curves (AUCs) ranging from 0.83 to 0.96 (Fig. 11). 
Overall, tumor hemodynamic parameters exhibited significantly high accuracies for early prediction of clinical outcomes. Such predications, based on frequent optical measurements, may ultimately be used to optimize individual therapeutic outcomes at an early time of therapy. For example, treatment outcomes may be improved by dynamically promoting oxygenation levels (e.g., hyperbaric oxygen therapy) in HPV-16 positive tumors or inhibiting angiogenesis (e.g., anti-VEGF antibody) in HPV-16 negative tumors.

\section{Summary and future perspectives}

In contrast to large imaging modalities such as CT, MRI, and PET, optical instruments such as NIRS/DOS/DOT and DCS/DCT are portable, fast, inexpensive, and suitable for continuous measurements at the bedside of clinical settings. NIRS/DOS/DOT techniques have been extensively used to measure tissue oxygenation in the clinic. Compared to NIRS/DOS/DOT, DCS/DCT is a relatively new technique that enables direct measurement of tissue blood flow. DCS/DCT or hybrid DCS/NIRS is being increasingly employed worldwide in a large variety of clinical contexts for the diagnosis and therapeutic monitoring of various diseases affecting tissue hemodynamics and metabolism. Overall, these pilot clinical studies in relatively small populations have shown the technique's versatility and demonstrated that the method provides new and complementary information about patient pathophysiology in a noninvasive fashion. It should be noticed that DCS/DCT has only been applied to a small population of patients with short measurement time frames (minutes to several weeks). Longitudinal studies in large populations are needed to elucidate potential unresolved technical problems and translate DCS/DCT into the clinic as a routine diagnostic and monitoring tool. 
There has been a concern on the physical modeling of blood flow (i.e., the motion of red blood cells) in the microvasculature. It was found that diffusive motion (see Section 2.1) fits the experimental autocorrelation curves rather well over a broad range of tissue types (Durduran, 2004; Zhou, 2007). Intuitively, however, random ballistic flow would be considered as a better model to fit the DCS/DCT data. Modified mixture models were proposed to include both ballistic flow and diffusive motion (Carp et al., 2011; Boas et al., 2016). Experimental data supported the proposed models for capturing the transition from early ballistic to subsequent diffusive motion. Computer simulations on the tissue with varied vessel diameters and spacing showed that the diffusive motion dominates the correlation decay in typical DCS measurements, and the blood flow index is modulated proportionally by the concentration of hemoglobin and the average diameter of blood vessels. Nevertheless, more clinical investigations are needed for accurate extracting of blood flow information in deep tissues.

Some technical issues exist when applying NIR diffuse optical technologies to deep tissues with heterogeneous properties. Since NIRS and DCS techniques rely on the transport of NIR light through top layer tissues (e.g., skin, skull), both methods must account for partial volume contributions from the top layer-structure to the deep tissue signals (e.g., brain, muscle, and tumor). In addition, measurement signal-to-noise ratio (SNR) and penetration depth are two interrelated and important parameters that affect the utility of both DCS and NIRS. Furthermore, most research with DCS reports only relative changes of blood flow index with respect to some baseline condition. 
The "partial volume effect" substantially affects the accuracy of DCS measurements, as discussed in literature (Durduran and Yodh, 2014; Yu, 2012a; Strangman et al., 2003). Slab-layered models were proposed to reduce the partial volume effect (Jaillon et al., 2006; Li et al., 2005; Verdecchia et al., 2016). A recently developed method, "Modified Beer-Lambert law for blood flow", has been proved to be effective in pressure modulation experiments to reduce the skin-effect on cerebral blood flow measurements (Baker et al., 2014; Baker et al., 2015). However, those methods ignored the influence of irregular tissue geometries. Recently, we created a new algorithm integrating a linear model of autocorrelation function with the Monte Carlo simulation of photon migrations in heterogeneous tissues with arbitrary geometries for simultaneous extraction of blood flow indices in multiple layered tissues (Shang and $\mathrm{Yu}, 2014)$.

Compared to NIRS/DOS/DOT, DCS/DCT measurements have relatively lower SNRs when probing deep tissue blood flow with large S-D separations (e.g., >2.5 mm). This is due to the utilization of single-mode detector fibers with a small core diameter of $5 \mu \mathrm{m}$ in DCS/DCT measurements to ensure the detection of autocorrelation functions of light intensity. Efforts have been made to improve SNR through the spatial average of multiple autocorrelation functions detected by a detector fiber bundle (Dietsche et al., 2007) or the temporal average of DCS signals obtained by a fast software correlator (Wang et al., 2016).

A potential remedy for the absolute blood flow measurement problem is to calibrate DCS/DCT against a gold standard. As mentioned early, comparison studies in small populations have been done in skeletal muscles, tumors, and brains against other established methods including power spectral Doppler ultrasound (Yu et al., 2005b), Doppler ultrasound 
(Buckley et al., 2009; Roche-Labarbe et al., 2010), laser Doppler (Durduran, 2004; Shang et al., 2011a), Xenon-CT (Kim et al., 2010), fluorescent microsphere flow measurement (Zhou et al., 2009), and ASL-MRI (Yu et al., 2007). Results from these studies show the promise to obtain absolute flow measurements although further validation works need to be done with different types of tissues in large populations.

DCT/ncDCT enables 3-D imaging of blood flow distributions in deep tissues. While effective, ncDCS/ncDCT employs a limited number of expensive APDs for blood flow detection, leading to low spatiotemporal resolution and high instrument cost (Lin et al., 2014; He et al., 2015; Huang et al., 2015b). With current ncDCT, the ROI must be mechanically scanned for a complete tissue coverage, which can take up to 40 minutes and may result in motion artifacts. To overcome these limitations, we recently developed and tested a non-scanning, noncontact, fast, portable, cost-effective device, namely speckle contrast diffuse correlation tomography ( $\mathrm{scDCT}$ ), for 3-D imaging flow distributions (Huang et al., 2015a; Huang et al., 2016). The scDCT used a charge-coupled-device (CCD) as a 2-D detector array to cover a ROI, thus eliminating the mechanical scanning and consequent problems. Thousands of detectors provided by the CCD significantly improved spatiotemporal resolution and reduced instrument cost/size. The scDCT prototype has been tested using computer simulations and tissue phantoms with anomaly flow contrast design. We are currently translating this novel optical technique to the clinic.

It is well known that many diseases are associated with tissue hypoxia, which is influenced by oxygen supply (i.e., blood flow) and tissue oxygen consumption. Simultaneous measurements of tissue blood flow and blood oxygenation using combined DCS and NIRS 
instruments enable the evaluation of tissue metabolic rate of oxygen consumption (Durduran et al., 2004; Roche-Labarbe et al., 2010; Lin et al., 2016; Dehaes et al., 2014; Durduran et al., 2010b; Buckley et al., 2013; Henry et al., 2015; Shang et al., 2012; Gurley et al., 2012; Chung et al., 2015). This metabolic parameter is potentially a more direct indicator of tissue metabolic activities, which integrates many factors and provides further insight about tissue pathophysiology. It is expected that with further technology development and more clinical applications, DCS/DCT and hybrid DCS/NIRS technologies will be eventually utilized as routine diagnostic and intervention monitoring tools for clinical investigations of various diseases.

\section{Acknowledgement}

We acknowledge support from the National Institutes of Health (NIH) R01-CA149274, R21-AR062356, and UL-1RR033173 Pilot Grant. We also acknowledge support of Research Funds from the American Heart Association Grant-In-Aid \#16GRNT30820006, National Endowment for Plastic Surgery Grant 3048112770, National Science Foundation (NSF) 1539068, and the National Key Research and Development Program of China 2016YFC0101600. The content herein is solely the responsibility of the authors and does not necessarily represent the official views of NIH and other foundations. 
References:

Al-Rawi P G and Kirkpatrick P J 2006 Tissue oxygen index - Thresholds for cerebral ischemia using near-infrared spectroscopy Stroke 37 2720-5

Arridge S R and Hebden J C 1997 Optical imaging in medicine: II. Modelling and reconstruction Phys Med Biol 42 841-53

Arridge S R and Lionheart W R 1998 Nonuniqueness in diffusion-based optical tomography Opt Lett 23 882-4

Baker W B, Parthasarathy A B, Busch D R, Mesquita R C, Greenberg J H and Yodh A G 2014 Modified Beer-Lambert law for blood flow Biomed. Opt. Express 5 4053-75

Baker W B, Parthasarathy A B, Ko T S, Busch D R, Abramson K, Tzeng S Y, Mesquita R C, Durduran T, Greenberg J H, Kung D K and Yodh A G 2015 Pressure modulation algorithm to separate cerebral hemodynamic signals from extracerebral artifacts Neurophotonics 2035004

Boas D A 1996 Diffuse Photon Probes of Structural and Dynamical Properties of Turbid Media: Theory and Biomedical Applications University of Pennsylvania: Philadelphia, USA pp 1-244

Boas D A, Campbell L E and Yodh A G 1995 Scattering and imaging with diffusing temporal field correlations Phys. Rev. Lett. 75 1855-8

Boas D A, Gaudette T, Strangman G, Cheng X F, Marota J J A and Mandeville J B 2001 The accuracy of near infrared spectroscopy and imaging during focal changes in cerebral hemodynamics Neuroimage 13 76-90

Boas D A, Sakadzic S, Selb J, Farzam P, Franceschini M A and Carp S A 2016 Establishing the diffuse correlation spectroscopy signal relationship with blood flow Neurophotonics 3031412

Boas D A and Yodh A G 1997 Spatially varying dynamical properties of turbid media probed with diffusing temporal light correlation Journal of the Optical Society of America a-Optics Image Science and Vision 14 192-215

Bouye P, Jacquinandi V, Picquet J, Thouveny F, Liagre J, Leftheriotis G, Saumet J L and Abraham P 2005 Near-infrared spectroscopy and transcutaneous oxygen pressure during exercise to detect arterial ischemia at the buttock level: Comparison with arteriography J. Vasc. Surg. 41 994-9

Brown W 1993 Dynamic Light Scattering: The Method and Some Applications Clarendon: New York

Buckley E M, Cook N M, Durduran T, Kim M N, Zhou C, Choe R, Yu G, Shultz S, Sehgal C M, Licht D J, Arger P H, Putt M E, Hurt H and Yodh A G 2009 Cerebral hemodynamics in preterm infants during positional intervention measured with diffuse correlation spectroscopy and transcranial Doppler ultrasound Opt. Express 17 12571-81

Buckley E M, Lynch J M, Goff D A, Schwab P J, Baker W B, Durduran T, Busch D R, Nicolson S C, Montenegro L M, Naim M Y, Xiao R, Spray T L, Yodh A G, Gaynor J W and Licht D J 2013 Early postoperative changes in cerebral oxygen metabolism following neonatal cardiac surgery: Effects of surgical duration Journal of Thoracic and Cardiovascular Surgery 145 196-205 
Busch D R, Lynch J M, Winters M E, McCarthy A L, Newland J J, Ko T, Cornaglia M A, Radcliffe J, McDonough J M, Samuel J, Matthews E, Xiao R, Yodh A G, Marcus C L, Licht D J and Tapia I E 2016a Cerebral Blood Flow Response to Hypercapnia in Children with Obstructive Sleep Apnea Syndrome Sleep 39 209-16

Busch D R, Rusin C G, Miller-Hance W, Kibler K, Baker W B, Heinle J S, Fraser C D, Yodh A G, Licht D J and Brady K M 2016b Continuous cerebral hemodynamic measurement during deep hypothermic circulatory arrest Biomed. Opt. Express 7 3461-70

Busch T M, Hahn S M, Evans S M and Koch C J 2000 Depletion of tumor oxygenation during photodynamic therapy: detection by the hypoxia marker EF3 [2-(2-nitroimidazol-1[H]-yl)-N-(3,3,3-trifluoropropyl)acetamide ] Cancer Res 60 $2636-42$

Carlson D J 2006 Mechanisms of Intrinsic Radiation Sensitivity: The Effects of DNA Damage Repair, Oxygen, and Radiation Quality In School of Health Sciences Purdue University: West Lafayette, IN, USA

Carp S A, Roche-Labarbe N, Franceschini M A, Srinivasan V J, Sakadzic S and Boas D A 2011 Due to intravascular multiple sequential scattering, Diffuse Correlation Spectroscopy of tissue primarily measures relative red blood cell motion within vessels Biomed. Opt. Express 2 2047-54

Cheng R 2013 Noninvasive near-infrared diffuse optical monitoring of cerebral hemodynamics and autoregulation University of Kentucky: Lexington, Kentucky, USA pp 1-121

Cheng R, Shang Y, Hayes D, Saha S P and Yu G 2012 Noninvasive optical evaluation of spontaneous low frequency oscillations in cerebral hemodynamics Neuroimage 62 1445-54

Cheng R, Shang Y, Wang S Q, Evans J M, Rayapati A, Randall D C and Yu G 2014 Near-infrared diffuse optical monitoring of cerebral blood flow and oxygenation for the prediction of vasovagal syncope J. Biomed. Opt. 19017001

Cheung C, Culver J P, Takahashi K, Greenberg J H and Yodh A G 2001 In vivo cerebrovascular measurement combining diffuse near-infrared absorption and correlation spectroscopies Phys. Med. Biol. 46 2053-65

Choe R 2005 Diffuse Optical Tomography and Spectroscopy of Breast Cancer and Fetal Brain University of Pennsylvania: Philadelphia, USA pp 1-226

Choe R, Putt M E, Carlile P M, Durduran T, Giammarco J M, Busch D R, Jung K W, Czerniecki B J, Tchou J, Feldman M D, Mies C, Rosen M A, Schnall M D, DeMichele A and Yodh A G 2014 Optically Measured Microvascular Blood Flow Contrast of Malignant Breast Tumors Plos One 9 e99683

Chung S H, Feldman M D, Martinez D, Kim H, Putt M E, Busch D R, Tchou J, Czerniecki B J, Schnall M D, Rosen M A, DeMichele A, Yodh A G and Choe R 2015 Macroscopic optical physiological parameters correlate with microscopic proliferation and vessel area breast cancer signatures Breast Cancer Res 1772

Cordero M D, De Miguel M, Fernandez A M M, Lopez I M C, Maraver J G, Cotan D, Izquierdo L G, Bonal P, Campa F, Bullon P, Navas P and Alcazar J A S 2010 Mitochondrial dysfunction and mitophagy activation in blood mononuclear cells of 
fibromyalgia patients: implications in the pathogenesis of the disease Arthritis. Res. Ther 12 R17

Culver J P, Durduran T, Furuya T, Cheung C, Greenberg J H and Yodh A G 2003 Diffuse optical tomography of cerebral blood flow, oxygenation, and metabolism in rat during focal ischemia J. Cereb. Blood Flow Metab. 23 911-24

Dehaes M, Aggarwal A, Lin P Y, Fortuno C R, Fenoglio A, Roche-Labarbe N, Soul J S, Franceschini M A and Grant P E 2014 Cerebral oxygen metabolism in neonatal hypoxic ischemic encephalopathy during and after therapeutic hypothermia J. Cereb. Blood Flow Metab. 34 87-94

Dehaes M, Cheng H H, Buckley E M, Lin P Y, Ferradal S L, Williams K, Vyas R, Hagan K, Wigmore D, McDavitt E, Soul J S, Franceschini M A, Newburger J W and Grant P E 2015 Perioperative cerebral hemodynamics and oxygen metabolism in neonates with single-ventricle physiology Biomed. Opt. Express 6 4749-67

Dehghani H, Eames M E, Yalavarthy P K, Davis S C, Srinivasan S, Carpenter C M, Pogue B W and Paulsen K D 2009 Near infrared optical tomography using NIRFAST: Algorithm for numerical model and image reconstruction Commun Numer Meth En 25 711-32

Dietsche G, Ninck M, Ortolf C, Li J, Jaillon F and Gisler T 2007 Fiber-based multispeckle detection for time-resolved diffusing-wave spectroscopy: characterization and application to blood flow detection in deep tissue Appl. Optics $\mathbf{4 6}$ 8506-14

Dong J, Bi R Z, Ho J H, Thong P S P, Soo K C and Lee K 2012a Diffuse correlation spectroscopy with a fast Fourier transform-based software autocorrelator J. Biomed. Opt. 17097004

Dong L 2015 Diffuse optical measurements of head and neck tumor hemodynamics for early prediction of chemo-radiation therapy outcomes University of Kentucky: Lexington, Kentucky, USA pp 1-117

Dong L, Kudrimoti M, Cheng R, Shang Y, Johnson E L, Stevens S D, Shelton B J and Yu G 2012b Noninvasive diffuse optical monitoring of head and neck tumor blood flow and oxygenation during radiation delivery Biomed. Opt. Express 3 259-72

Dong L, Kudrimot M, Irwin D, Chen L, Kumar S, Shang Y, Huang C, Johnson E L, Stevens S D, Shelton B J and Yu G 2016 Diffuse optical measurements of head and neck tumor hemodynamics for early prediction of chemo-radiation therapy outcomes $J$. Biomed. Opt. 21085004

Durduran T 2004 Non-invasive measurements of tissue hemodynamics with hybrid diffuse optical methods University of Pennsylvania: Philadelphia, USA pp 1-208

Durduran T, Choe R, Baker W B and Yodh A G 2010a Diffuse optics for tissue monitoring and tomography Rep. Prog. Phys. 73076701

Durduran T, Choe R, Yu G, Zhou C, Tchou J C, Czerniecki B J and Yodh A G 2005 Diffuse optical measurement of blood flow in breast tumors Opt. Lett. 30 2915-7

Durduran T and Yodh A G 2014 Diffuse correlation spectroscopy for non-invasive, micro-vascular cerebral blood flow measurement Neuroimage 85 51-63

Durduran T, Yu G, Burnett M G, Detre J A, Greenberg J H, Wang J J, Zhou C and Yodh A G 2004 Diffuse optical measurement of blood flow, blood oxygenation, and metabolism in a human brain during sensorimotor cortex activation Opt. Lett. 29 1766-8 
Durduran T, Zhou C, Edlow B L, Yu G, Choe R, Kim M N, Cucchiara B L, Putt M E, Shah Q, Kasner S E, Greenberg J H, Yodh A G and Detre J A 2009 Transcranial optical monitoring of cerebrovascular hemodynamics in acute stroke patients Opt. Express 17 3884-902

Durduran T, Zhou C A, Buckley E M, Kim M N, Yu G, Choe R, Gaynor J W, Spray T L, Durning S M, Mason S E, Montenegro L M, Nicolson S C, Zimmerman R A, Putt M E, Wang J J, Greenberg J H, Detre J A, Yodh A G and Licht D J 2010b Optical measurement of cerebral hemodynamics and oxygen metabolism in neonates with congenital heart defects J. Biomed. Opt. 15037004

Eggebrecht A T, Ferradal S L, Robichaux-Viehoever A, Hassanpour M S, Dehghani H, Snyder A Z, Hershey T and Culver J P 2014 Mapping distributed brain function and networks with diffuse optical tomography Nat Photonics 8 448-54

Fantini S, Franceschinifantini M A, Maier J S, Walker S A, Barbieri B and Gratton E 1995 Frequency-Domain Multichannel Optical-Detector for Noninvasive Tissue Spectroscopy and Oximetry Optical Engineering 34 32-42

Favilla C G, Mesquita R C, Mullen M, Durduran T, Lu X P, Kim M N, Minkoff D L, Kasner S E, Greenberg J H, Yodh A G and Detre J A 2014 Optical Bedside Monitoring of Cerebral Blood Flow in Acute Ischemic Stroke Patients During Head-of-Bed Manipulation Stroke 45 1269-74

Ferrari M, Muthalib M and Quaresima V 2011 The use of near-infrared spectroscopy in understanding skeletal muscle physiology: recent developments Philosophical Transactions of the Royal Society a-Mathematical Physical and Engineering Sciences 369 4577-90

Fletcher G C 1976 Dynamic light scattering from collagen solutions. I. Translational diffusion coefficient and aggregation effects Biopolymers 15 2201-17

Franceschini M A, Joseph D K, Huppert T J, Diamond S G and Boas D A 2006 Diffuse optical imaging of the whole head J. Biomed. Opt. 11054007

Gagnon L, Desjardins M, Jehanne-Lacasse J, Bherer L and Lesage F 2008 Investigation of diffuse correlation spectroscopy in multi-layered media including the human head Opt. Express 16 15514-30

Ghosh A, Elwell C and Smith M 2012 Cerebral Near-Infrared Spectroscopy in Adults: A Work in Progress Anesth Analg 115 1373-83

Grassi W, Core P, Carlino G, Salaffi F and Cervini C 1994 Capillary-Permeability in Fibromyalgia Journal of Rheumatology 21 1328-31

Gurley K 2012 Use of hybrid diffuse optical spectroscopies in continuous monitoring of blood flow, blood oxygenation and oxygen consumption rate in exercising skeletal muscle University of Kentucky: Lexington, Kentucky, USA pp 1-81

Gurley K, Shang Y and Yu G 2012 Noninvasive optical quantification of absolute blood flow, blood oxygenation, and oxygen consumption rate in exercising skeletal muscle $J$. Biomed. Opt. 17075010

He L 2015 Noncontact diffuse correlation tomography of breast tumor University of Kentucky: Lexington, Kentucky, USA pp 1-126

He L, Lin Y, Huang C, Irwin D, Szabunio M M and Yu G 2015 Noncontact diffuse correlation tomography of human breast tumor J. Biomed. Opt. 2086003 
Henry B, Zhao M J, Shang Y, Uhl T, Thomas D T, Xenos E S, Saha S P and Yu G 2015 Hybrid diffuse optical techniques for continuous hemodynamic measurement in gastrocnemius during plantar flexion exercise J. Biomed. Opt. 20125006

Hou Y, Shang Y, Cheng R, Zhao Y, Qin Y, Kryscio R, Rayapati A, Hayes D and Yu G 2014 Obstructive sleep apnea-hypopnea results in significant variations in cerebral hemodynamics detected by diffuse optical spectroscopies Physiol. Meas. 35 2135-48

Huang C, Irwin D, Lin Y, Shang Y, He L, Kong W K, Luo J and Yu G 2015a Speckle contrast diffuse correlation tomography of complex turbid medium flow Med Phys $\mathbf{4 2}$ 4000-6

Huang C, Lin Y, He L, Irwin D, Szabunio M M and Yu G 2015b Alignment of sources and detectors on breast surface for noncontact diffuse correlation tomography of breast tumors Appl. Optics $\mathbf{5 4} 8808-16$

Huang C, Radabaugh J P, Aouad R K, Lin Y, Gal T J, Patel A B, Valentino J, Shang Y and Yu G 2015c Noncontact diffuse optical assessment of blood flow changes in head and neck free tissue transfer flaps J. Biomed. Opt. 20075008

Huang C, Seong M, Morgan J P, Mazdeyasna S, Kim J G, Hastings J T and Yu G 2016 A low-cost compact diffuse speckle contrast flowmeter using small laser diode and bare charge-coupled-device J. Biomed. Opt. 21080501

Intes X, Chen J, Venugopal V and Lesage F 2010 Time-resolved diffuse optical tomography with patterned-light illumination and detection Opt. Lett. 35 2121-3

Irwin D 2011 Influence of tissue absorption and scattering on diffuse correlation spectroscopy blood flow measurement University of Kentucky: Lexington, Kentucky, USA pp $1-68$

Irwin D, Dong L, Shang Y, Cheng R, Kudrimoti M, Stevens S D and Yu G 2011 Influences of tissue absorption and scattering on diffuse correlation spectroscopy blood flow measurements Biomed. Opt. Express 2 1969-85

Jaillon F, Li J, Dietsche G, Elbert T and Gisler T 2007 Activity of the human visual cortex measured non-invasively by diffusing-wave spectroscopy Opt. Express 15 6643-50

Jaillon F, Skipetrov S E, Li J, Dietsche G, Maret G and Gisler T 2006 Diffusing-wave spectroscopy from head-like tissue phantoms: influence of a non-scattering layer Opt. Express 14 10181-94

Jermyn M, Ghadyani H, Mastanduno M A, Turner W, Davis S C, Dehghani H and Pogue B W 2013 Fast segmentation and high-quality three-dimensional volume mesh creation from medical images for diffuse optical tomography J. Biomed. Opt. 1886007

Jobsis F F 1977 Noninvasive, infrared monitoring of cerebral and myocardial oxygen sufficiency and circulatory parameters Science 198 1264-7

Kasikcioglu E, Dinler M and Berker E 2006 Reduced tolerance of exercise in fibromyalgia may be a consequence of impaired microcirculation initiated by deficient action of nitric oxide Medical Hypotheses 66 950-2

Kim J G, Xia M N and Liu H L 2005 Extinction coefficients of hemoglobin for near-infrared spectroscopy of tissue IEEE Eng. Med. Biol. Mag. 24 118-21

Kim M N, Durduran T, Frangos S, Edlow B L, Buckley E M, Moss H E, Zhou C, Yu G, Choe R, Maloney-Wilensky E, Wolf R L, Grady M S, Greenberg J H, Levine J M, Yodh A G, Detre J A and Kofke W A 2010 Noninvasive measurement of cerebral blood flow 
and blood oxygenation using near-infrared and diffuse correlation spectroscopies in critically brain-Injured adults Neurocrit. Care 12 173-80

Kim M N, Edlow B L, Durduran T, Frangos S, Mesquita R C, Levine J M, Greenberg J H, Yodh A G and Detre J A 2014 Continuous Optical Monitoring of Cerebral Hemodynamics During Head-of-Bed Manipulation in Brain-Injured Adults Neurocrit. Care 20 443-53

Li J, Dietsche G, Iftime D, Skipetrov S E, Maret G, Elbert T, Rockstroh B and Gisler T 2005 Noninvasive detection of functional brain activity with near-infrared diffusing-wave spectroscopy J. Biomed. Opt. 1044002

Li T, Lin Y, Shang Y, He L, Huang C, Szabunio M and Yu G 2013 Simultaneous measurement of deep tissue blood flow and oxygenation using noncontact diffuse correlation spectroscopy flow-oximeter Sci. Rep. 31358

Lin P Y, Hagan K, Fenoglio A, Grant P E and Franceschini M A 2016 Reduced cerebral blood flow and oxygen metabolism in extremely preterm neonates with low-grade germinal matrix-intraventricular hemorrhage Sci. Rep. 625903

Lin Y, He L, Shang Y and Yu G 2012 Noncontact diffuse correlation spectroscopy for noninvasive deep tissue blood flow measurement J. Biomed. Opt. 17010502

Lin Y, Huang C, Irwin D, He L, Shang Y and Yu G 2014 Three-dimensional flow contrast imaging of deep tissue using noncontact diffuse correlation tomography Applied Physics Letters 104121103

Lindh M, Johansson G, Hedberg M, Henning G B and Grimby G 1995 Muscle-Fiber Characteristics, Capillaries and Enzymes in Patients with Fibromyalgia and Controls Scandinavian Journal of Rheumatology 24 34-7

Liu H, Boas D A, Zhang Y, Yodh A G and Chance B 1995 Determination of optical properties and blood oxygenation in tissue using continuous NIR light Phys Med Biol 40 1983-93

Maret G and Wolf P E 1987 Multiple Light-Scattering from Disordered Media - the Effect of Brownian-Motion of Scatterers Zeitschrift Fur Physik B-Condensed Matter 65 409-13

Maret G and Wolf P E 1989 Multiple Light-Scattering - Weak Localization and Dynamic Fluctuations Physica Scripta T29 223-5

McIver K L, Evans C, Kraus R M, Ispas L, Sciotti V M and Hickner R C 2006 NO-mediated alterations in skeletal muscle nutritive blood flow and lactate metabolism in fibromyalgia Pain 120 161-9

Mesquita R C, Durduran T, Yu G, Buckley E M, Kim M N, Zhou C, Choe R, Sunar U and Yodh A G 2011 Direct measurement of tissue blood flow and metabolism with diffuse optics Philosophical Transactions of the Royal Society a-Mathematical Physical and Engineering Sciences 369 4390-406

Mesquita R C, Putt M, Chandra M, Yu G, Xing X M, Han S W, Lech G, Shang Y, Durduran T, Zhou C, Yodh A G and Mohler E R 2013 Diffuse optical characterization of an exercising patient group with peripheral artery disease J. Biomed. Opt. 1857007

Morf S, Amann-Vesti B, Forster A, Franzeck U K, Koppensteiner R, Uebelhart D and Sprott H 2005 Microcirculation abnormalities in patients with fibromyalgia - measured by capillary microscopy and laser fluxmetry Arthritis. Res. Ther 7 R209-R16

Munk N, Symons B, Shang Y, Cheng R and Yu G 2012 Noninvasively measuring the 
hemodynamic effects of massage on skeletal muscle: A novel hybrid near-infrared diffuse optical instrument Journal of Bodywork and Movement Therapies 16 22-8

Murkin J M and Arango M 2009 Near-infrared spectroscopy as an index of brain and tissue oxygenation British Journal of Anaesthesia 103 (BJA/PGA Supplement) i3-i13

Patterson M S, Chance B and Wilson B C 1989 Time resolved reflectance and transmittance for the non-invasive measurement of tissue optical properties Appl Opt 28 2331-6

Pine D J, Weitz D A, Chaikin P M and Herbolzheimer E 1988 Diffusing-Wave Spectroscopy Phys. Rev. Lett. 60 1134-7

Pine D J, Weitz D A, Zhu J X and Herbolzheimer E 1990 Diffusing-Wave Spectroscopy Dynamic Light-Scattering in the Multiple-Scattering Limit Journal De Physique 51 2101-27

Quaresima V, Ferrari M, Franceschini M A, Hoimes M L and Fantini S 2004 Spatial distribution of vastus lateralis blood flow and oxyhemoglobin saturation measured at the end of isometric quadriceps contraction by multichannel near-infrared spectroscopy J. Biomed. Opt. 9 413-20

Rice S O 1954 Mathematical analysis of random noise In Noise and Stochastic Processes Wax N ed Dover: New York p 133

Roche-Labarbe N, Carp S A, Surova A, Patel M, Boas D A, Grant R E and Franceschini M A 2010 Noninvasive optical measures of $\mathrm{CBV}, \mathrm{StO}(2), \mathrm{CBF}$ Index, and $\mathrm{rCMRO}(2)$ in human premature neonates' brains in the first six weeks of life Hum. Brain Mapp. 31 $341-52$

Schachner T, Bonaros N, Bonatti J and Kolbitsch C 2008 Near infrared spectroscopy for controlling the quality of distal leg perfusion in remote access cardiopulmonary bypass Eur J Cardiothorac Surg 34 1253-4

Schweiger M and Arridge S 2014 The Toast plus plus software suite for forward and inverse modeling in optical tomography J. Biomed. Opt. 19

Shang Y, Chen L, Toborek M and Yu G 2011a Diffuse optical monitoring of repeated cerebral ischemia in mice Opt. Express 19 20301-15

Shang Y, Cheng R, Dong L, Ryan S J, Saha S P and Yu G 2011b Cerebral monitoring during carotid endarterectomy using near-infrared diffuse optical spectroscopies and electroencephalogram Phys. Med. Biol. 56 3015-32

Shang Y, Gurley K, Symons B, Long D, Srikuea R, Crofford L J, Peterson C A and Yu G 2012 Noninvasive optical characterization of muscle blood flow, oxygenation, and metabolism in women with fibromyalgia Arthritis. Res. Ther 14 R236

Shang Y and Yu G 2014 A Nth-order linear algorithm for extracting diffuse correlation spectroscopy blood flow indices in heterogeneous tissues Applied Physics Letters 105

Shang Y, Zhao Y, Cheng R, Dong L, Irwin D and Yu G 2009 Portable optical tissue flow oximeter based on diffuse correlation spectroscopy Opt. Lett. 34 3556-8

Shuler M S, Reisman W M, Whitesides T E, Kinsey T L, Hammerberg E M, Davila M G and Moore T J 2009 Near-Infrared Spectroscopy in Lower Extremity Trauma Journal of Bone and Joint Surgery-American Volume 91A 1360-8

Strangman G, Franceschini M A and Boas D A 2003 Factors affecting the accuracy of near-infrared spectroscopy concentration calculations for focal changes in oxygenation parameters Neuroimage 18 865-79 
Sunar U, Quon H, Durduran T, Zhang J, Du J, Zhou C, Yu G, Choe R, Kilger A, Lustig R, Loevner L, Nioka S, Chance B and Yodh A G 2006 Noninvasive diffuse optical measurement of blood flow and blood oxygenation for monitoring radiation therapy in patients with head and neck tumors: a pilot study J. Biomed. Opt. 11064021

Vardi M and Nini A 2008 Near-infrared spectroscopy for evaluation of peripheral vascular disease. A systematic review of literature Eur. J. Vasc. Endovasc. Surg. 35 68-74

Verdecchia K, Diop M, Lee A, Morrison L B, Lee T Y and St Lawrence K 2016 Assessment of a multi-layered diffuse correlation spectroscopy method for monitoring cerebral blood flow in adults Biomed. Opt. Express 7 3659-74

Wang D T, Parthasarathy A B, Baker W B, Gannon K, Kavuri V, Ko T, Schenkel S, Li Z, Li Z R, Mullen M T, Detre J A and Yodh A G 2016 Fast blood flow monitoring in deep tissues with real-time software correlators Biomed. Opt. Express 7 776-97

Wolf M, Ferrari M and Quaresima V 2007 Progress of near-infrared spectroscopy and topography for brain and muscle clinical applications J. Biomed. Opt. 12062104

Wolf M, Franceschini M A, Paunescu L A, Toronov V, Michalos A, Wolf U, Gratton E and Fantini S 2003 Absolute frequency-domain pulse oximetry of the brain: methodology and measurements Adv Exp Med Biol 530 61-73

Yu G 2012a Diffuse Correlation Spectroscopy (DCS): A Diagnostic Tool for Assessing Tissue Blood Flow in Vascular-Related Diseases and Therapies Current Medical Imaging Reviews 8 194-210

Yu G 2012b Near-infrared diffuse correlation spectroscopy (DCS) for assessing deep tissue blood flow Anatomy and Physiology 2 1000e115

Yu G 2012c Near-infrared diffuse correlation spectroscopy in cancer diagnosis and therapy monitoring J. Biomed. Opt. 17010901

Yu G, Durduran T, Lech G, Zhou C, Chance B, Mohler E R and Yodh A G 2005a Time-dependent blood flow and oxygenation in human skeletal muscles measured with noninvasive near-infrared diffuse optical spectroscopies J. Biomed. Opt. 10 024027

Yu G, Durduran T, Zhou C, Wang H W, Putt M E, Saunders H M, Sehgal C M, Glatstein E, Yodh A G and Busch T M 2005b Noninvasive monitoring of murine tumor blood flow during and after photodynamic therapy provides early assessment of therapeutic efficacy Clin. Cancer. Res. 11 3543-52

Yu G, Floyd T F, Durduran T, Zhou C, Wang J J, Detre J A and Yodh A G 2007 Validation of diffuse correlation spectroscopy for muscle blood flow with concurrent arterial spin labeled perfusion MRI Opt. Express 15 1064-75

Yu G, Shang Y, Zhao Y, Cheng R, Dong L and Saha S P 2011 Intraoperative evaluation of revascularization effect on ischemic muscle hemodynamics using near-infrared diffuse optical spectroscopies J. Biomed. Opt. 16027004

Zhang W, Gao F, Wu L H, Ma W J, Lu Y M and Zhao H J 2013 A time-domain diffuse fluorescence and optical tomography system for breast tumor diagnosis $J$ Infrared Millim W 32 181-6

Zhou C 2007 In-Vivo Optical Imaging and Spectroscopy of Cerebral Hemodynamics University of Pennsylvania: Philadelphia, USA pp 1-325

Zhou C, Choe R, Shah N, Durduran T, Yu G, Durkin A, Hsiang D, Mehta R, Butler J, Cerussi 
A, Tromberg B J and Yodh A G 2007 Diffuse optical monitoring of blood flow and oxygenation in human breast cancer during early stages of neoadjuvant chemotherapy J. Biomed. Opt. 12051903

Zhou C, Eucker S A, Durduran T, Yu G, Ralston J, Friess S H, Ichord R N, Margulies S S and Yodh A G 2009 Diffuse optical monitoring of hemodynamic changes in piglet brain with closed head injury J. Biomed. Opt. 14034015

Zhou C, Yu G, Furuya D, Greenberg J H, Yodh A G and Durduran T 2006 Diffuse optical correlation tomography of cerebral blood flow during cortical spreading depression in rat brain Opt. Express 14 1125-44

Zirak P, Delgado-Mederos R, Dinia L, Carrera D, Marti-Fabregas J and Durduran T 2014 Transcranial diffuse optical monitoring of microvascular cerebral hemodynamics after thrombolysis in ischemic stroke J. Biomed. Opt. 1918002 


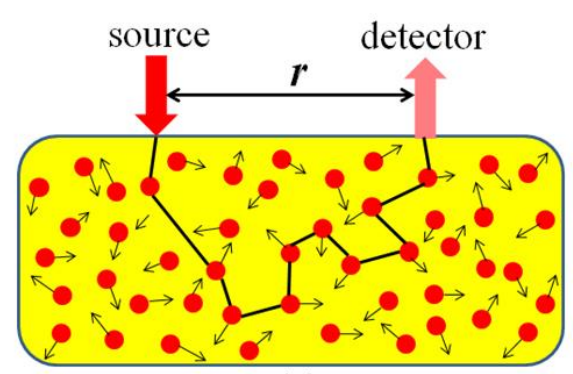

(a)

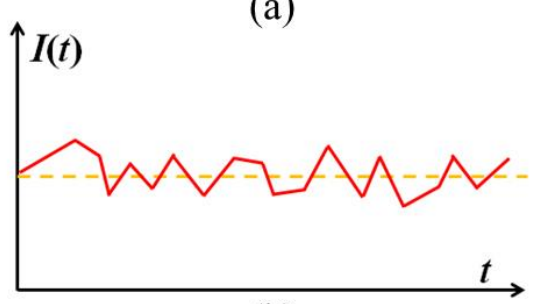

(b)

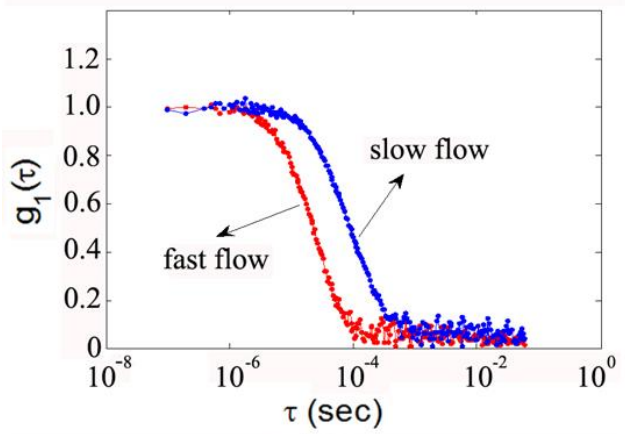

(c)

Fig. 1: The principle of diffuse correlation spectroscopy (DCS) for blood flow measurements in deep/thick tissues. (a) Source and detector fibers are placed on the tissue surface within a distance of $\mathbf{r}$ for light delivery and collection; (b) Light intensity $\mathrm{I}(\mathrm{t})$, detected by the APD, fluctuates with time due to the motions of RBCs; (c) Blood flow change can be quantified from the shift of temporal electric field autocorrelation function curves. 


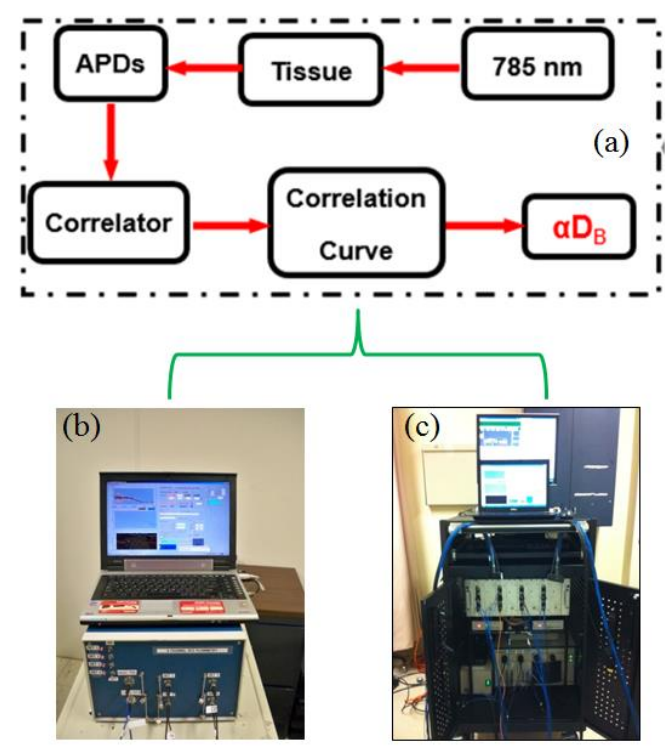

DCS

DCT

Fig. 2: The schematic diagram (a) and instrument photos of typical DCS (b) and DCT (c) systems. 


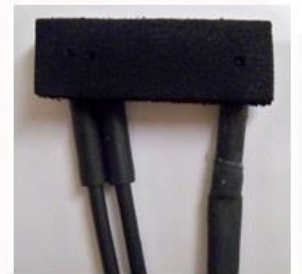

(a)

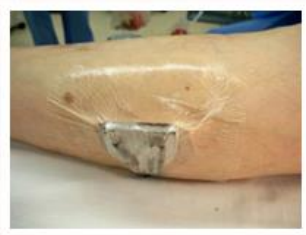

(c)

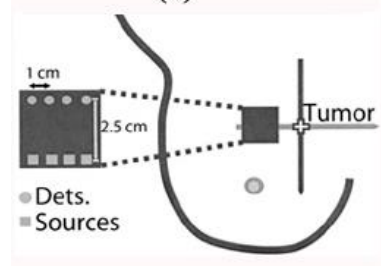

(d)

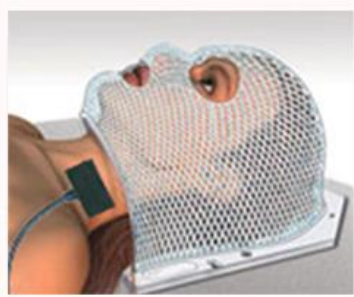

(e)

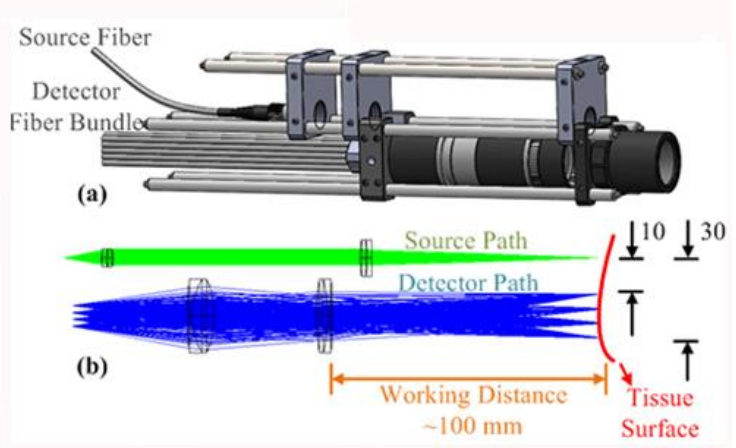

(f)

Fig. 3: A variety of optical probes for DCS/DCT measurements. (a) A typical DCS probe consisting of source and detector fibers; (b) Two DCS probes taped on both sides of a frontal head for cerebral blood flow measurements; (c) A DCS probe taped on top of the calf muscle; (d) A hand-held DCS probe scanning over a breast tumor; (e) A DCS probe placed on top of the head/neck tumor; (f) A noncontact DCS probe with lens system focusing the source and detectors on the surface of tissue. Some of the figures are reproduced from the subfigures in the references (Huang et al., 2015c; Durduran et al., 2005) 


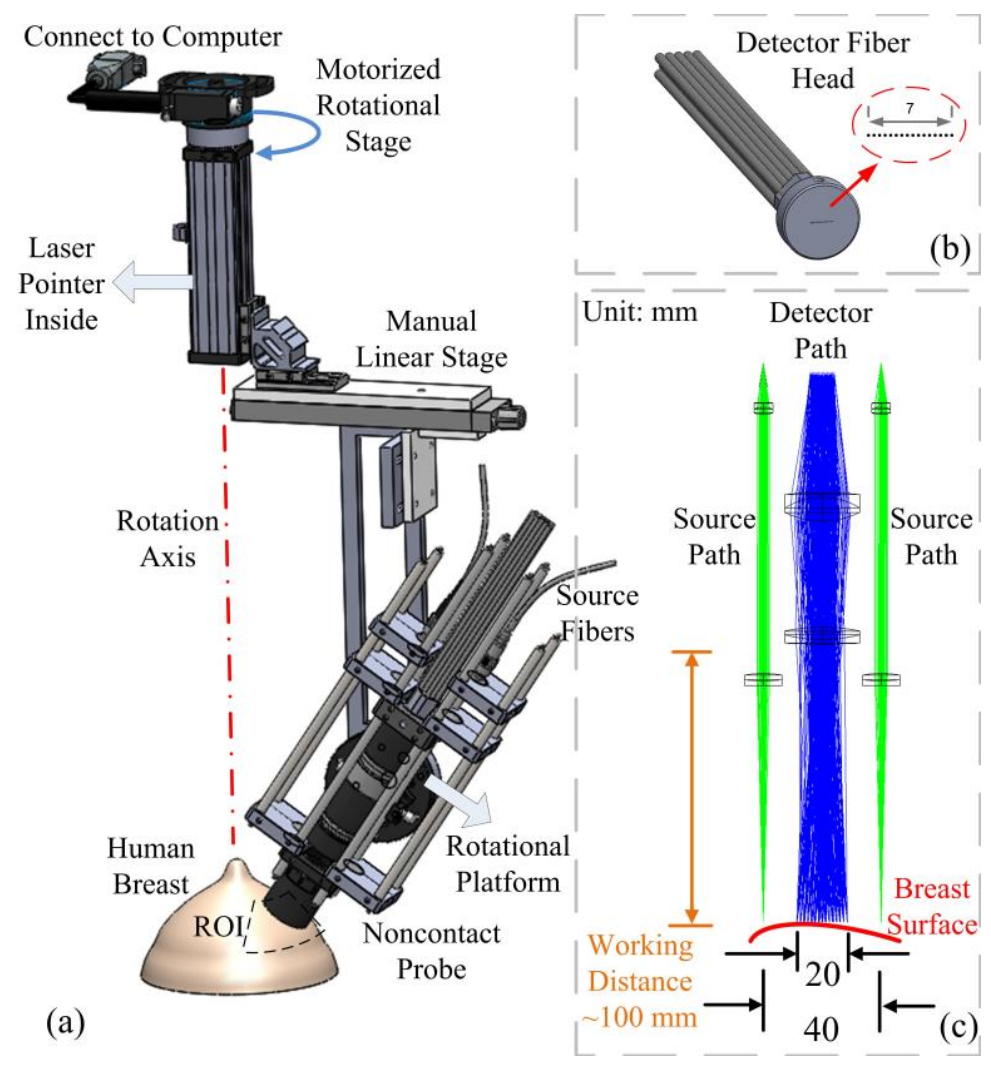

Fig. 4: A noncontact DCT probe with rotational scanning system. (a) Noncontact DCT probe head was scanned over a region of interest (ROI) on the breast by a motorized rotational stage, (b) A linear array of single-mode detector fibers, (c) Source and detector fibers were projected on the breast surface using achromatic lenses; two source paths were attached to the sides of the detector path. This figure is reproduced from Fig. 1 in the reference (Huang et al., 2015b). 


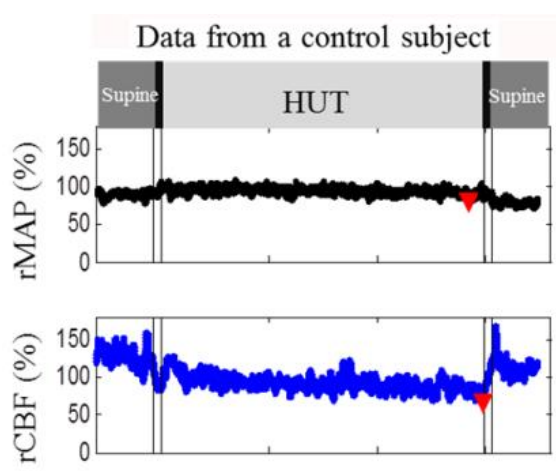

(a)

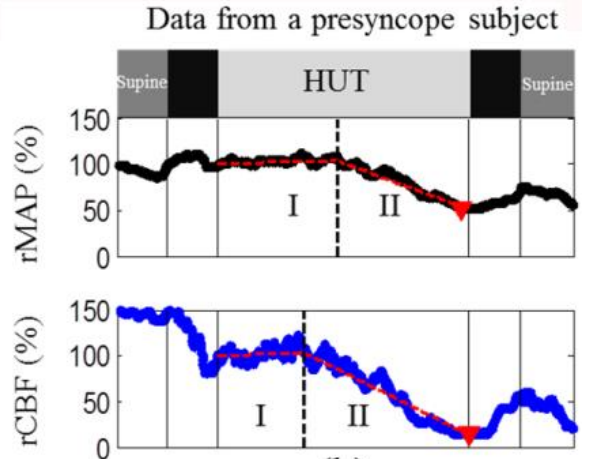

(b)

Fig. 5. Relative changes in mean artery blood pressure (rMAP) and cerebral blood flow (rCBF) from a control subject (a) and a presyncope subject (b) during Head-Up-Tilting (HUT). The solid vertical lines indicate the beginning or ending of tilting up and tilting down. The triangles indicate the minima of rMAP and rCBF during HUT. The two connected dashed lines on top of the raw data (b) demonstrate the two-line fitting results. The dashed vertical lines indicate the break-points separating Stage I and Stage II. This figure is reproduced from the subfigures of Fig. 1 in the reference (Cheng et al., 2014). 


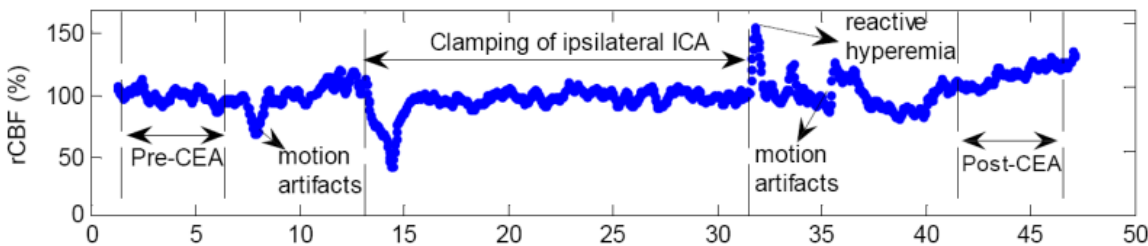

(a)

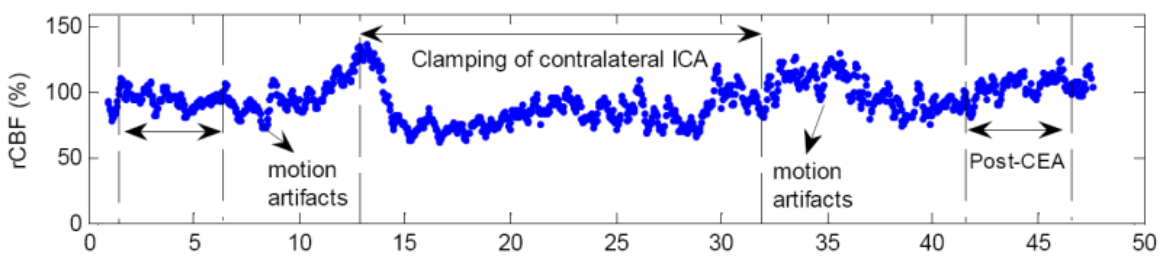

(b)

Fig. 6. Typical relative changes in cerebral blood flow (rCBF) (a) at the surgical side (ipsilateral) and (b) at the nonsurgical side (contralateral). This figure is reproduced from subfigures of Fig. 2 in the reference (Shang et al., 2011b). 

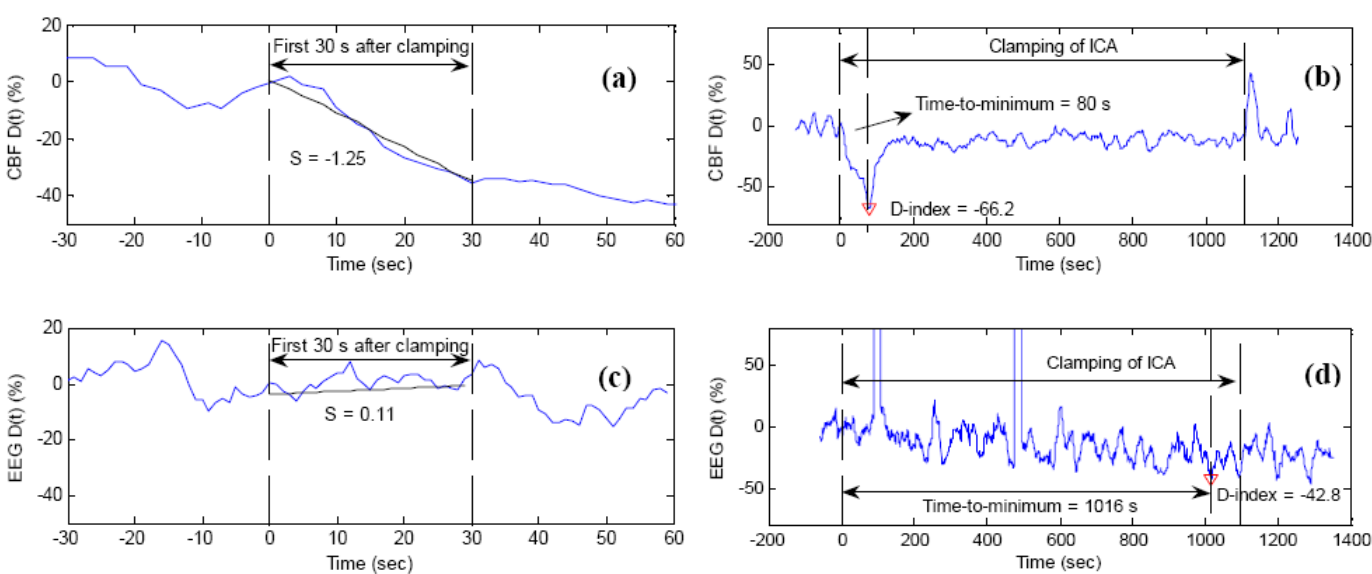

Fig. 7: Comparison of DCS and EEG measurement results in a typical patient during carotid endarterectomy (CEA) when the internal carotid artery (ICA) was temporarily clamped. The resulted ischemic status by the ICA clamping was characterized by the slope (S), $D$-index, and time-to-minimum for both CBF (top panel) and EEG (bottom panel) at the surgical side. Here the slope (S) is the deceasing rate of variable $D(t)$ during the first 30 -second clamping period, and $D(t)$ represents the percentage change of variable (CBF or EEG spectral power) at time $t$, when compared to pre-clamping baseline. The minimum of $D(t)$ during the entire period of clamping is defined as $D$-index, representing the most severe cerebral hypoperfusion during ICA clamping. The period from the beginning of clamping to the time of $D(t)$ attaining its minimum ( $D$-index) is defined as time-to-minimum. The large negative CBF slope $(\mathrm{S}=-1.25)$ during the first 30 -second clamping period indicates the rapid CBF decrease due to ICA clamping (a). The time duration of CBF decrease and maximal CBF decrease during the entire clamping period were characterized by time-to-minimum (80 s) and $D$-index (-66.2\%), respectively (b). By contrast, the EEG power changes were small and slow $(\mathrm{S}=0.11)(\mathbf{c})$, and reached its minimum $(D$-index $=-42.8 \%)$ in a long period of time (time-to-minimum $=1016 \mathrm{~s})(\mathbf{d})$. This figure is reproduced from Fig. 4 in the reference (Shang et al., 2011b). 
Fig. 8: Average absolute blood flow responses to handgrip exercise in 10 subjects with fibromyalgia (FM) and 13 age-matched healthy controls (HC). The vertical lines indicate the beginning and end of exercise. Average pre-exercise blood flow value, determined as the average of data points $30 \mathrm{~s}$ before the onset of exercise, was $3.19 \pm 1.03 \mathrm{ml} / 100 \mathrm{ml} / \mathrm{min}$ for the healthy subjects and $2.63 \pm 0.71 \mathrm{ml} / 100 \mathrm{ml} / \mathrm{min}$ for the FM subjects $(\mathrm{p}=0.444)$. Plateau blood flow value, determined as the average of points $30 \mathrm{~s}$ before the end of exercise was $10.71 \pm 3.91 \mathrm{ml} / 100 \mathrm{ml} / \mathrm{min}$ for healthy subjects and $4.83 \pm 1.42 \mathrm{ml} / 100 \mathrm{ml} / \mathrm{min}$ for $\mathrm{FM}$ subjects $(\mathrm{p}=0.018)$. 


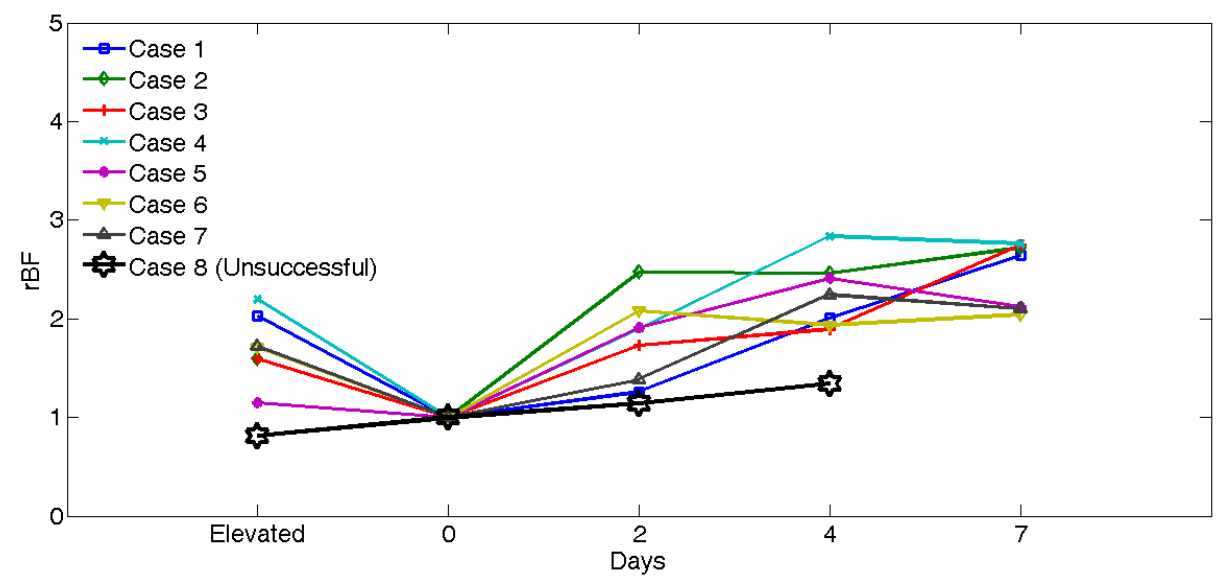

Fig. 9: Comparison of rBF changes between seven successful and one unsuccessful flaps. The rBF values over the seven successful cases (Cases 1 to 8 ) during operation and on postoperative days $2,4,7$ are presented individually. rBF from the unsuccessful flap case (Case 8) was measured at the time of flap elevation and on postoperative days 2 and 4 . The data from the unsuccessful case (Case 8) at Day 7 are not available due to the subsequent re-flap before that day. This figure is reproduced from Fig. 6 in the reference (Huang et al., 2015c). 
(a)

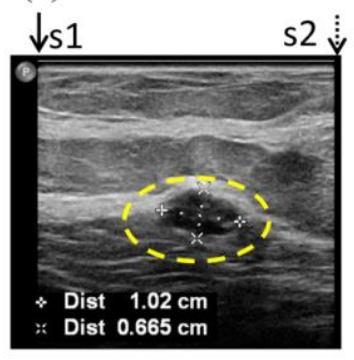

(d)

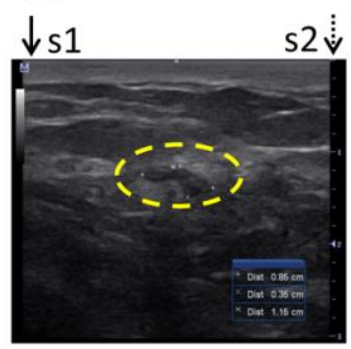

(b)

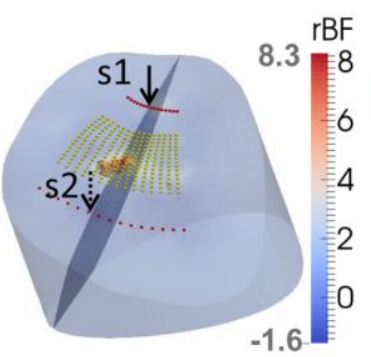

(e)

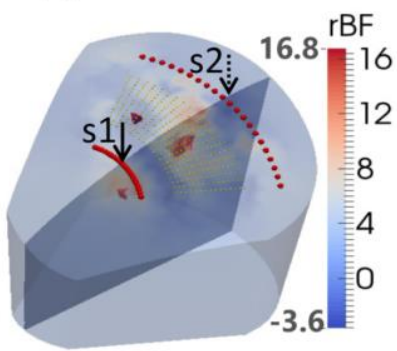

(c)

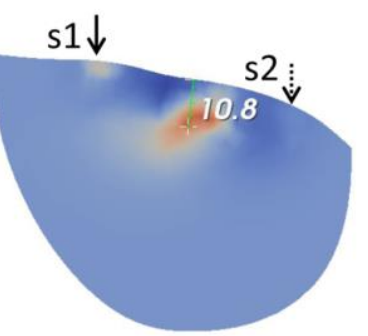

(f)

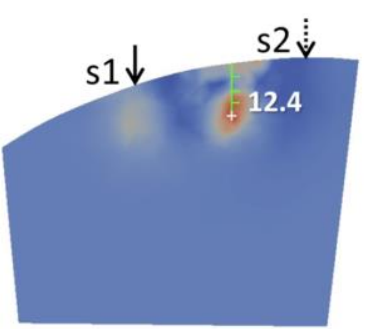

Fig. 10: Clinical examples of two low-grade carcinomas in situ. (a) Ultrasound image of breast with a tumor (inside the yellow dashed circle) in the first patient. The tumor mass center is located at $19.2 \mathrm{~mm}$ beneath the skin surface. (d) Ultrasound image of breast with a tumor (inside the yellow dashed circle) in the second patient. The tumor mass center is located at $13.3 \mathrm{~mm}$ beneath the skin surface. (b) and (e) show the reconstructed 3-D tumor blood flow contrasts imaged by the ncDCT in the first and second patients, respectively. For the comparison of ultrasound and ncDCT results, an ultrasound imaging plane along the transducer line and across the overlapped two specific light sources (S1 and S2) of ncDCT (see Fig. 4) is presented in the 3-D reconstructed image. The backgrounds are presented with $30 \%$ transparency of the original color clarity. (c) and (f) show the cross-section views through the reconstructed tumor centers, which can be directly compared to the 2-D ultrasound tumor images $[(\mathbf{a})$ and $(\mathbf{d})]$, respectively. This figure is reproduced from Fig. 8 in the reference (He et al., 2015). 


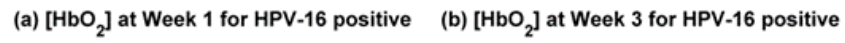
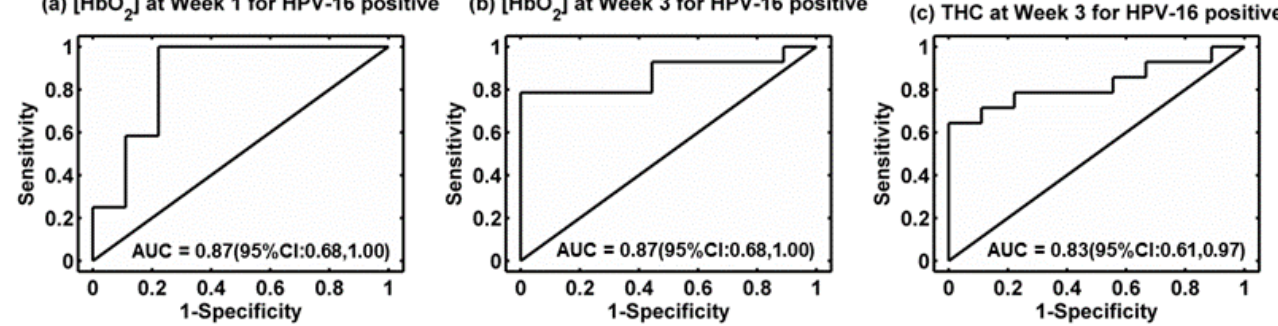

(d) $\mathrm{StO}_{2}$ at Week 3 for HPV-16 positive
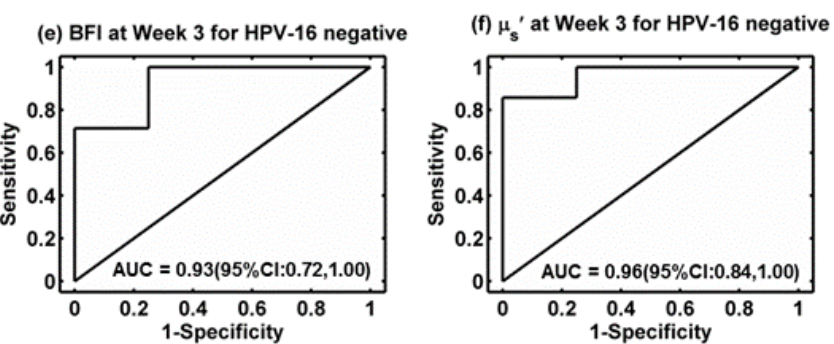

Fig. 11: Receiver operating characteristic curves (ROC) and the associated areas under the curve (AUC) for discriminating tumors with incomplete response (IR) or complete response (CR). (a, b) $\left[\mathrm{HbO}_{2}\right]$ at Week 1 and Week 3 in HPV-16 positive patients, (c) THC at Week 3 in HPV-16 positive patients, (d) $\mathrm{StO}_{2}$ at Week 3 in HPV-16 positive patients, (e) BFI in HPV-16 negative patients at Week 3, and (f) $\mu_{\mathrm{s}}$ ' in HPV-16 negative patients at Week 3. This figure is reproduced from Fig. 5 in the reference (Dong et al., 2016). 